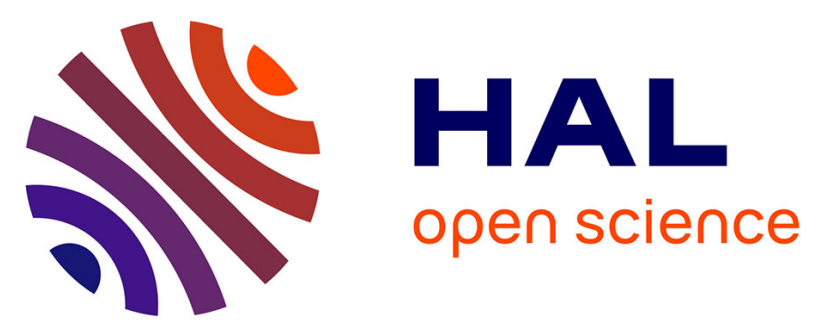

\title{
Pour la mort d'un chef kanak. Le destin de Mohamed ben Ahmed ou les enjeux d'une histoire coloniale des subalternes. La Nouvelle-Calédonie au tournant du XXe siècle
}

Isabelle Merle, Adrian Muckle

\section{To cite this version:}

Isabelle Merle, Adrian Muckle. Pour la mort d'un chef kanak. Le destin de Mohamed ben Ahmed ou les enjeux d'une histoire coloniale des subalternes. La Nouvelle-Calédonie au tournant du XXe siècle. Revue d'Histoire Moderne et Contemporaine, 2017, 2017/3 (n 64-3), pp.9-38. 10.3917/rhmc.643.0009 . halshs-01422677

\section{HAL Id: halshs-01422677 \\ https://shs.hal.science/halshs-01422677}

Submitted on 30 Dec 2021

HAL is a multi-disciplinary open access archive for the deposit and dissemination of scientific research documents, whether they are published or not. The documents may come from teaching and research institutions in France or abroad, or from public or private research centers.
L'archive ouverte pluridisciplinaire HAL, est destinée au dépôt et à la diffusion de documents scientifiques de niveau recherche, publiés ou non, émanant des établissements d'enseignement et de recherche français ou étrangers, des laboratoires publics ou privés. 


\section{Pour la mort d'un chef kanak. Le destin de Mohamed ben Ahmed ou les enjeux d'une histoire coloniale des subalternes. La Nouvelle-Calédonie au tournant du $\mathrm{xx}^{\mathrm{e}}$ siècle}

Isabelle MERLE

Adrian MUCKLE

Le 24 septembre 2009 fut commémorée à Koné, siège de la Province Nord en Nouvelle-Calédonie, la mort du chef kanak Bwëé Noël Pwatiba, figure héroïque de l'insurrection kanak qui éclata en 1917, dans le nord calédonien, à l'ombre de la Grande Guerre. Organisée par l'association Töémirî popai ("entretenir la parole") avec le soutien de la mairie, du conseil culturel de la province Nord et la participation des districts coutumiers de Poindah et de Baco, l'initiative fit événement en pays kanak car, pour la première fois, pouvaient s'exprimer au grand jour des paroles évoquant les souvenirs traumatiques de la "guerre de 17", ultime insurrection kanak d'un long XIX ${ }^{\mathrm{e}}$ siècle colonial, inscrite dans la lignée de celles qui s'opposèrent à la conquête française depuis 1853, et qui toucha surtout la région nord de l'île.

Dans un livre récent, Les sanglots de l'aigle pêcheur, Alban Bensa, Yvon Goromoedo et Adrian Muckle relatent en détail la chronologie des faits, le contexte précis dans lequel ils prennent sens, l'épaisseur d'une histoire kanak, en opérant une percée remarquable sur les points de vue des acteurs, les mises en récit de l'époque ainsi que les constructions mémorielles ultérieures qui, aujourd'hui, s'expriment et se partagent ${ }^{1}$.

Nul n'ignorait en 2009 que le chef Bwëé Noël Pwatiba avait été tué par un "Arabe $»^{2}$ qui l'avait ensuite décapité pour apporter sa tête à la gendarmerie

1. On rappellera ici aussi «La guerre kanak de 1917», numéro spécial de Mwà Véé: revue culturelle kanak, 62, 2008. Ce qu'on appelle aujourd'hui la "guerre de 1917» est l'objet du livre d'Adrian MUCKLE, Specters of Violence in a Colonial Context: New Caledonia, 1917, Honolulu, University of Hawai'i Press, 2012. On se référera aussi à Sylvette BOUBIN-BOyer, De la Première Guerre mondiale en Océanie: les guerres de tous les calédoniens, vol. 2, Villeneuve d'Ascq, Presses universitaires du Septentrion, 2003.

2. Le terme "Arabe» ou "arabe», selon la typographie utilisée dans les archives, recouvre usuellement en Nouvelle-Calédonie les condamnés, transportés ou déportés issus des colonies françaises d'Afrique du 


\section{DOCUMent 1}

La «guerre de 17»

La «guerre de 17» peut être considérée comme le dernier événement d'une longue série d'insurrections kanak contre la colonisation française, qui commence dès 1853, date de la prise de possession de l'île par la France, et se déplace de lieux en lieux, en fonction de l'intensité de la pression coloniale, jusqu'à embraser en 1878 la région kanak riche et peuplée de La Foa/Fonwhary/Farino avec des ramifications au Sud jusqu'à Bouloupari et au nord dans l'arrière-pays de Koné. Communément appelée «l'insurrection kanak de 1878 ", il serait pourtant plus juste de parler de "guerre» car, du point de vue kanak, c'est bien la guerre qui est engagée contre les Blancs en 1878 comme en 1917.

Restée jusque-là relativement à l'écart du mouvement d'implantation européen, si ce n'est quelques éleveurs poussant leur bétail au mépris des cultures kanak, la région centre-nord est investie à partir de 1880 avec la création du centre de colonisation libre de Koné (1880), celle du centre pénal de Poumbout Koniambo (1883) puis du centre libre de Voh/Témala en 1892-1895.

Les Kanak du Nord n'échappent pas aux impositions de plus en plus lourdes d'une politique indigène qui organise progressivement le cantonnement généralisé des «tribus» dans des réserves indigènes (1867-1900) et qui applique à partir de 1887 le régime de l'indigénat avec la restriction des libertés de circulation, la multiplication des obligations de travail (corvées, réquisitions, prestations), la mise en place de l'impôt de capitation (1899). Ce contexte de prédation coloniale justifiée par un racisme idéologique constitue le terreau sur lequel prennent naissance les guerres kanak au cours du XIX ${ }^{\mathrm{e}}$ siècle, comme c'est le cas encore en 1917, date à laquelle s'ajoutent les effets de la mobilisation pour la Grande Guerre. La «guerre de 17》 s’inscrit dans la lignée de celle de 1878 par les "causes" qui la déterminent mais aussi par les pratiques: stratégies d'alliances, organisation, gestuelle, interprétations qui, en monde kanak, puisent dans le registre très ancien des guerres en y ajoutant les outils de la "modernité» : le fusil, l'usage des chevaux, l'observation aiguisée des techniques de combats déployées par les militaires français. L'effort, cependant, ne parviendra pas à renverser le rapport de force colonial déjà solidement installé. La "guerre de 17" ne prend pas l'ampleur de celle de 1878. Elle reste localisée en région nord et s'essouffle rapidement pour s'éteindre en 1918.

de Koné où elle a été enterrée. La violence du crime, l'absence de cérémonies coutumières et l'impossible deuil firent de la commémoration de 2009 un événement répondant à un double objectif: la réparation symbolique des rites funéraires qui n'avaient pu être accomplis en leurs temps et la délivrance d'un message politique appelant à une autre histoire coloniale nourrie par les multiples versions des acteurs impliqués, Kanak et non Kanak. Ainsi s'exprimait le maire de Koné ouvrant la cérémonie:

Nord, Algérie, Maroc, Tunisie. La majorité venait d'Algérie, dont les fameux déportés suite à la grande révolte de Kabylie en 1871 sous l'influence de la famille Mokrani. Les transportés issus des colonies françaises d'Afrique du Nord furent nombreux dans les convois qui arrivèrent entre 1894 et 1897, dont fait partie Mohamed ben Ahmed. Nous utilisons quant à nous la typographie "Arabe» pour marquer la distance avec la formulation de l'époque sauf lorsqu'il s'agit d'une citation. 


\section{Document 2}

Les guerres kanak en Nouvelle-Calédonie

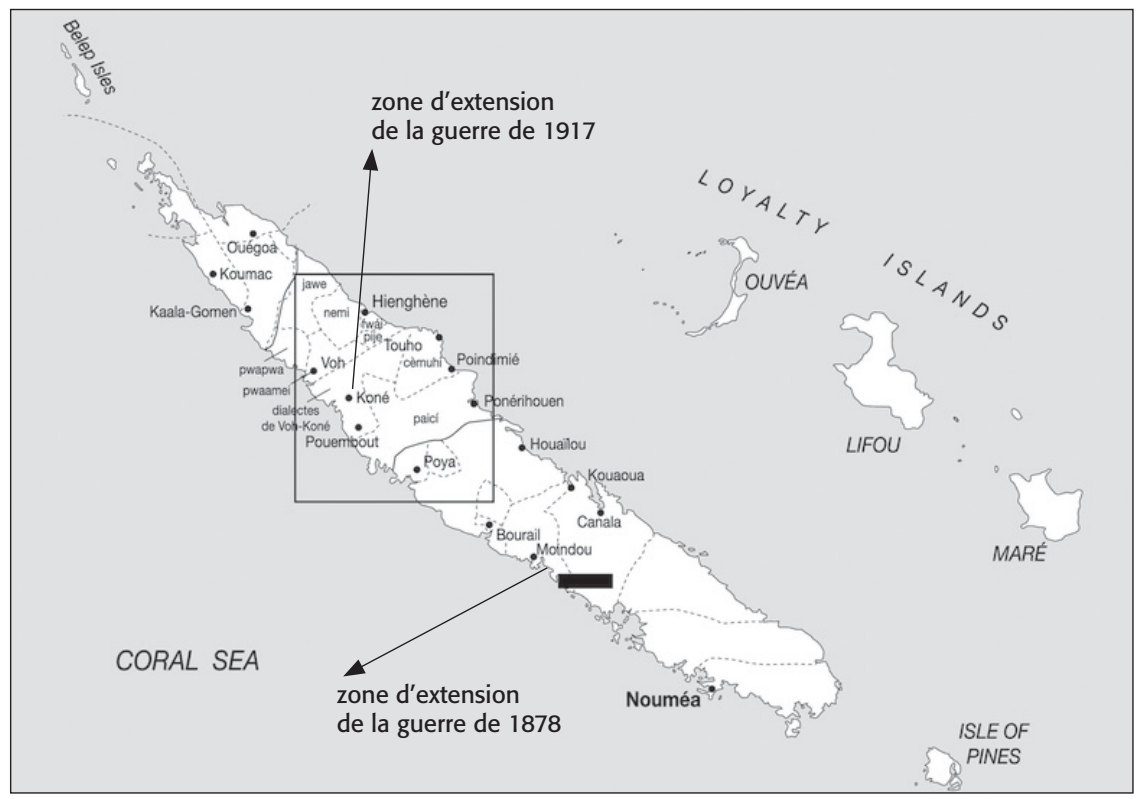

"Aujourd'hui 24 septembre 2009, on a refusé d'être les victimes de l'histoire coloniale. Ce jour, on doit relever la tête. Avec les autres communautés, on est ensemble pour marquer une nouvelle page de l'histoire. Inaugurer la stèle ${ }^{3}$, c'est donc une manière de faire notre coutume, les choses qui nous sont propres" ${ }^{4}$.

Si l'appel aux autres communautés ${ }^{5}$ est lancé, la cérémonie est cependant faite par et pour les Kanak et on comprend dans ce contexte que l'"Arabe» n'y ait alors aucune place. Il ne pouvait pas trouver toute sa place non plus dans le travail publié récemment sur «1917» par Alban Bensa et Adrian Muckle, fruit de recherches de longue haleine dont l'effort a surtout porté sur l'histoire des Kanak à l'épreuve de la colonisation plutôt que sur les autres acteurs en présence et les interactions entre communautés ${ }^{6}$.

3. Une stèle a été posée en mémoire du chef Noël et de son engagement dans l'insurrection.

4. Apégu Joseph GoROMIDO, allocution du 24 septembre 2009, citée in Alban BENSA, Kacué Yvon Goromoedo, A. MUCKLe, Les sanglots de l'aigle pêcheur. Nouvelle-Calédonie: la guerre kanak de 1917, Toulouse, Anacharsis, 2015, p. 195-196. Sur la commémoration: ibidem, p. 171-211.

5. La population de la Nouvelle-Calédonie est composée, selon le recensement de 2014, de 268767 personnes dont 40,3\% déclarent appartenir à la communauté kanak, 29,2\% à la communauté européenne, $8,7 \%$ à la communauté wallisienne et futunienne et $7,3 \%$ sont regroupés sous le terme «autre»: Tahitiens (2\%), Indonésiens (1,6\%), Vietnamiens, Ni-Vanuatu $(0,9 \%)$, autres asiatiques $(0,8 \%)$ et autres $(1 \%)$. Source: Insee, 2014.

6. A. Bensa, K.Y. Goromoedo, A. MUCKLe, Les sanglots..., op. cit., p. 182-184. 
Mohamed ben Ahmed, en ce sens, constitue l'aiguillon de l'enquête que nous voudrions présenter ici pour tenter de rendre à cet homme son épaisseur historique, sa trajectoire personnelle et le sens de ses actions. Nous voulons compléter en quelque sorte le dossier mais aussi ouvrir les pistes d'une réflexion en prenant le pari d'une histoire coloniale saisie "au ras du sol", une sorte de grounded subaltern colonial history à travers la trajectoire d'un homme et au gré de ses rencontres dans un lieu-dit niché sur la côte nord-ouest de la NouvelleCalédonie, au croisement de destins les plus divers d'individus happés par les dynamiques et connexions impériales - émigrants français attirés par la propagande et la promesse de terres, condamnés aux travaux forcés originaires de France, d'Algérie, du Maroc, d'Italie ou de Corse, auxquels il faut ajouter les travailleurs engagés sur contrat d'origine asiatique, Japonais, Javanais, Tonkinois ou autres - tous échoués dans la région de Koné-Pouembout au contact des Kanak progressivement repoussés dans les réserves indigènes.

En portant l'attention sur le cas de Mohamed ben Ahmed, son origine, sa traversée du bagne et son implantation dans le centre pénal de Koniambo, mais aussi sur les relations qu'il déploie, sa rencontre avec Noël, ses stratégies envers les colons de Koné et les officiels de la pénitentiaire, ses amitiés et trahisons, nous tentons d'éclairer au plus près et au plus juste les modalités pratiques du fonctionnement de certains aspects de la société coloniale en voie de constitution en Nouvelle-Calédonie. À travers la biographie d'un homme condamné à ce qu'on appelle alors «les travaux forcés» et l'étude minutieuse du contexte local dans lequel il s'inscrit et circule, il s'agit de mettre en œuvre une micro-sociologie historique capable de restituer les interactions les plus fines qui ont forgé la trame complexe du tissu social d'une colonie marquée par un double projet de colonisation, libre et pénal.

L'inspiration nous vient d'enquêtes historiques qui ont tenté cet effort descriptif très localisé pour saisir l'agency des acteurs les plus humbles dans les espaces impériaux et nous pensons d'abord à la célèbre étude de l'historien américain Richard White, consacrée à l'histoire de la rencontre entre Indiens et Européens, qu'il dénomme le Middle ground dans la région des grands lacs aux XVII ${ }^{\mathrm{e}}$ et XVIII ${ }^{\mathrm{e}}$ siècles ${ }^{7}$. Nous pourrions aussi évoquer le projet historiographique de l'école des Subaltern Studies indiennes tel qu'il fut développé dans les années 1980 et qui s'appuyait alors sur des case studies remarquablement contextualisées et documentées ${ }^{8}$.

Mais c'est indéniablement le livre de Clare Anderson, Subaltern Lives ${ }^{9}$, découvert au cours de l'enquête, qui se rapproche le plus de notre propos car

7. Richard White, Le Middle Ground. Indiens, Empires et République dans la région des grands lacs, 1650-1815 [1991], Toulouse, Anacharsis, 2009.

8. Isabelle MERLE, "Les Subaltern Studies. Retour sur les principes fondateurs d'un projet historiographique de l'Inde coloniale", Genèses, 56, 2004, p. 131-147.

9. Clare ANDERson, Subaltern Lives. Biographies of Colonialism in the Indian Ocean World, 17901920, Cambridge, Cambridge University Press, 2012. 
l'approche biographique utilisée par l'auteur retrace la trajectoire de convicts $^{10}$ saisis dans les mailles d'une histoire de la transportation britannique intracoloniale qui couvre divers points d'ancrages situés sur les rivages asiatiques de l'océan Indien jusqu'en Australie. Les Subaltern Lives que C. Anderson suit avec minutie touchent des hommes et des femmes de toutes origines, indiennes, africaines, eurasiennes, transportés vers l'île Maurice, vers les îles Andamans, à Van Diemen's Land ou vers la Nouvelle-Galles du Sud. Dans sa remarquable introduction, elle nous invite à reconsidérer les morceaux de vie de ces "subalternes» - méticuleusement reconstitués grâce aux archives lacunaires et fragmentaires qui peuvent y faire référence - comme des leviers de connaissance et de compréhension des processus et contextes qu'ils révèlent: la transportation et la fabrique de colonies pénales d'abord mais plus largement la formation des mondes coloniaux. Par une approche prosoprographique des subalternes, et en tout premier lieu des convicts, elle propose de mettre à l'épreuve la notion même de subalternité en travaillant "au ras du sol» et en contexte le fil de destins individuels croisés révélant la porosité des catégories coloniales autant que leur efficacité pour forger des univers sociaux singuliers.

L'histoire du forçat Mohamed ben Ahmed, d'origine marocaine, condamné en Algérie, transporté en Nouvelle-Calédonie puis placé sur concession rurale dans le centre pénal de Koniambo, au cœur de la région de Koné, a été pour nous une clé d'entrée et un levier pour tenter d'approcher au plus près ce que Georges Balandier appelait «la situation coloniale». L'enjeu, pour nous, est moins de s'intéresser aux processus de la transportation pénale en NouvelleCalédonie en tant que tels - quoiqu'il soit pourtant nécessaire de les évoquer - que de tenter de décrypter ce qui se joue à l'abri des regards officiels, dans un coin de vallée calédonienne, entre Mohamed et ceux qui l'entourent, les colons libres, les colons pénaux, les libérés, les Kanak et les autres, dans les interactions les plus quotidiennes, forcées ou consenties, entre ces hommes (et femmes) que le destin a placés là, sommés de se lier, pour le meilleur ou pour le pire, dans un contexte dominé par les rapports de force et les violences d'un système colonial et pénal.

C'est au prix de cet effort de reconstitution avec, en main, la clé que nous offre le cas de Mohamed appuyé par un travail rigoureux de recoupements d'archives diverses et éparpillées, que nous cherchons à sonder les raisons d'un crime et plus largement la nature des «relations coloniales» dans cette colonie française du Pacifique au tournant du $\mathrm{XX}^{\mathrm{e}}$ siècle. Ce sont, tapies dans l'ombre, ces questions jusque-là inavouées qui provoquaient l'émotion des acteurs de la commémoration à laquelle nous avons assisté, Adrian Muckle et moi-même, en 2009, devant le centre culturel de Koné, et auxquelles nous voulons apporter une tentative de réponse.

10. Terme anglais qualifiant les condamnés à la transportation. 


\section{LA MORT DE NOËL}

Le 10 janvier 1918, le journal quotidien La France Australe publie un télégramme reçu à 14 heures selon lequel «le chef rebelle Noël pourchassé par le Grandchef Noël de Bourail et nos partisans indigènes ${ }^{11}$ a été tué par un Arabe et un assigné ${ }^{12}$ dans une concession de Koniambo ${ }^{13}$. Le lendemain, 11 janvier, ce même journal donne plus de détails:

«Noël s'était rendu hier matin à 10 heures à Tiamou à l'habitation du concessionnaire Mohamed ben Ahmed auquel il demande des allumettes; il était exténué et mourant de faim, il ne portait pas d'armes. L'arabe lui remit 8 boîtes d'allumettes, lui offrit du café et du pain qu'il absorba avec avidité. Il déclara que ses deux frères étaient loin. Sa collation terminée, Noël se dirigea vers la porte pour reprendre la brousse. Mohamed le suivit et, arrivé à quelques pas de la maison, lui tira cinq coups de revolver. Quoique gravement blessé le chef rebelle s'enfuit et réussit à gagner le bord de la rivière situé à environ 50 mètres de l'habitation. L'arabe le rejoignit et un corps à corps terrible s'engagea dans lequel Noël fut terrassé après avoir reçu quelques coups de faucille bien appliqués. À ce moment le nommé Ducamp ${ }^{14}$ travaillant non loin du lieu de la lutte accourut, porteur d'un sabre d'abattis qu'il remit à Mohamed. Ce dernier s'en servit immédiatement pour décapiter Noël $»^{15}$.

Le même Mohamed ben Ahmed confirme les faits et précise lors d'un interrogatoire mené en mars 1918 à la gendarmerie de Koné :

«[Noël] est parti dans la direction de la rivière et en remontant vers Tiamou. Je l'ai suivi le revolver à la main et j'ai tiré sur lui près du creek; se sentant touché, il s'est écrié "Ah! Salaud!" [...]. Il n'était porteur d'aucune arme, pas même de bâton »"${ }^{16}$.

Une fois le forfait accompli, la tête fut mise dans un sac et transportée au poste de Koné.

\section{Le contexte d'une guerre}

Mohamed ben Ahmed vient de tuer Bwëé Noël Pwatiba, l'une des principales figures des actions guerrières que mènent des groupes kanak de la région Nord depuis avril 1917. Les affrontements ont commencé suite à une rencontre,

11. Ont été créés, à partir de 1898, des «Grands-chefs» responsables de districts indigènes avec, sous leurs ordres, des "petits chefs" responsables chacun du territoire et de la population de leur "tribu". Le terme de "partisans indigènes" renvoit ici aux forces auxiliaires indigènes qui ont précocement assisté les troupes françaises. Le Grand-chef de Bourail s'engage auprès des militaires pour soutenir la répression contre les «rebelles» du Nord.

12. "L'arabe et l'assigné» sont tous deux condamnés et placés sur concessions pénales à Koniambo.

13. La France Australe, 10 janvier 1918.

14. Ducamp, à l'évidence, est là par hasard. C'est un voisin de Mohamed, condamné pour de multiples vols en 1877 à Marseille et transporté en Nouvelle-Calédonie en 1878 où il est à nouveau condamné à plus de 70 ans de travaux forcés pour vols et évasions! Placé sur concession à une date inconnue, Ducamp obtient finalement sa remise de peine de vingt ans le 29 octobre 1919, suite peut-être à l'aide qu'il apporte à Mohamed pour décapiter Noël. Libérable en 1924, Ducamp meurt à Koniambo en 1923. Archives nationales d'outre-mer, Aix-en-Provence, fonds ministériels (désormais ANOM, FM) $\mathrm{H} / 1362$.

15. La France Australe, 11 janvier 1918.

16. Archives de Nouvelle-Calédonie, Nouméa (désormais ANC), 1W1, Médoux (gendarme), procès-verbal, Koné, 8 mars 1918. 
organisée par le chef du Service des affaires indigènes, qui était censée réconcilier deux parties en conflit, les hommes menés par Noël des lieux-dits Tiamou, Pana et Pwënaki et les alliés du petit chef Doui de Koniambo. Noël refuse de s'y rendre car il soupçonne à juste titre un piège tendu par l'administration coloniale. Ses guerriers narguant les soldats du haut de la colline, des coups de fusils sont échangés, des participants sont arrêtés et l'incident enflamme la région sur la côte ouest puis la côte est au cours de l'année ${ }^{17}$.

Les raisons de la guerre sont multiples et s'inscrivent dans l'épaisseur d'un passé immédiat ou plus lointain auquel les Kanak se réfèrent alors pour agir ou non, s'engager auprès "des rebelles", rester neutres ou au contraire partir avec les colonnes françaises comme auxiliaires. Le souvenir des guerres passées entre Kanak et les litiges anciens, l'expérience des humiliations et répressions exercées par les forces armées lors de la guerre de conquête, les spoliations des terres au profit de la colonisation, le bétail nouvellement introduit qui détruit les cultures, les interdictions de circulation et les réquisitions de travail liées à l'application du régime de l'indigénat depuis 1887 mais aussi le poids de l'impôt de capitation appliqué à partir de 1900 aux seuls Kanak ou la mise à l'écart obligatoire des lépreux décrétée dans les années 1910, sont autant d'éléments qui expliquent, en fonction de circonstances très localisées, les motivations à l'aune desquelles se forgent les décisions de "partir en guerre» ou non contre les Français.

S'ajoute à cela le contexte très tendu de la guerre de 14-18, avec l'engagement supposément volontaire des Kanak parmi les indigènes de l'Empire, décrété en 1915 et appliqué à partir de 1916 en Nouvelle-Calédonie par un premier mouvement de recrutement renouvelé en janvier 1917. L'engagement du chef Noël et des hommes qui l'entourent est directement lié à la pression qu'exerce le petit chef de la réserve de Koniambo, Doui, pour remplir le quota d'hommes à recruter, sachant que nombre de Kanak ne veulent pas s'engager dans la "guerre des blancs». Noël et Poindet Apengou (de Paola Netchaot) ont reçu des menaces directes du chef du service des Affaires indigènes, Fourcade, qui s'adresse à eux avec mépris, en "petit nègre", pour leur enjoindre de trouver des volontaires. Selon le gendarme Faure, Poindet Apengou s'emporte en lui répondant: "Mais laissez-nous tranquilles, nous ne sommes pas des cochons" tandis que Noël reste le visage fermé au point d'inquiéter Faure qui déclare que Fourcade ne s'est pas fait un $\mathrm{ami}^{18}$.

Explicitées en ces termes généraux, les causes de la guerre kanak de 1917 sont aisées à saisir. Cependant, c'est en réduisant la focale sur Koniambo en particulier et sur l'histoire de destins individuels que nous essayons de mieux

17. Pour plus de détails sur cet événement qui a eu lieu le 28 avril 1917 à Tiamou: A. MUCKLE, Specters..., op. cit., p. 51-57; A. BENSA, K.Y. GoROMOEDO, A. MUCKLE, Les sanglots..., op. cit., p. 50-72.

18. Brigadier FAURE [prénom non indiqué], «"Affaires de Koné”. Rapport du Brigadier Faure sur les débuts de l'insurrection de 1917 en Nouvelle-Calédonie", Fournal de la Société des Océanistes, 39-76, 1983, p. 69-88, p. 70; voir A. MUCKLE, Specters..., op. cit., p. 42. 
comprendre la genèse et les dynamiques dans lesquelles interagissent Kanak et Européens, pour éclairer un univers colonial «au ras du sol».

\section{"L'AGENCY SUBALTERNE »: CONCESSIONNAIRES PÉNAUX VERSUS KANAK OU L'HISTOIRE DES DEUX KONIAMBO}

Noël avait ses terres à Tiamou, lieu-dit kanak situé sur la réserve indigène de Koniambo. Du moins est-ce là un lieu refuge occupé par sa famille originaire de Poya, plus au sud, et déjà déplacée avec d'autres, suite aux répressions menées dans cette région lors de la grande révolte kanak de 1878. Les limites de la réserve de Koniambo, dessinées dans les années 1880, furent plus strictement fixées en 1900 à quelques kilomètres seulement des concessions pénales du centre pénitentiaire de Koniambo, lui-même créé en 1884. Chacun de ces lieux, la réserve indigène de Koniambo et le centre de colonisation pénale du même nom, sont le produit d'histoires institutionnelles autonomes ${ }^{19}$. Mais l'originalité du paysage social de la Nouvelle-Calédonie coloniale du début du $\mathrm{XX}^{\mathrm{e}}$ siècle et de la région de Koné, en particulier, se trouve précisément dans une confrontation inédite entre des populations kanak d'un côté et une population "blanche" très bigarrée de l'autre (auxquelles il faut ajouter les engagés sur contrat d'origines diverses mais massivement asiatiques, Javanais, Tonkinois ou Japonais).

La population «blanche» compte alors en son sein non seulement des colons dits libres, émigrants venus de leur propre gré, placés sur des terres dans des centres de colonisation "libres", mais aussi des condamnés aux travaux forcés transportés dans la colonie depuis 1863, devenus concessionnaires pénaux ou simplement libérés ${ }^{20}$ à l'issue de leur peine. À leur arrivée en Nouvelle-Calédonie, les transportés sont d'abord regroupés dans des pénitenciers (pénitencier principal à l'île Nou et près de Nouméa ou pénitenciers secondaires en brousse) ou sur des chantiers de route et dans les mines pour «effectuer les travaux

19. Sur l'origine des réserves indigènes en Nouvelle-Calédonie, voir par exemple I. MERLE, "De l'idée de cantonnement à la constitution des réserves. La définition de la propriété indigène", in A. BENSA, Isabelle LEBLIC (éd.), En pays Kanak. Ethnologie, linguistique, archéologie, histoire de la Nouvelle-Calédonie, Paris, Éditions de la Maison des sciences de l'homme, 2000, p. 217-234. Pour l'histoire de la colonisation pénale: EAD., Expériences coloniales. La Nouvelle-Calédonie (1853-1920), Paris, Belin, 1995. Sur la fondation du centre pénal de Pouembout: Cynthia DeBIEN, «La colonisation pénale en Nouvelle-Calédonie: l'exemple des concessionnaires de Pouembout 1883-1895", mémoire de maîtrise, Toulouse-Le Mirail, 1990.

20. On appelle «libérés» en Nouvelle-Calédonie les condamnés qui sortent du bagne sans avoir obtenu de concessions de terres. Cette formule est juridiquement inexacte au sens ou elle devrait recouvrir l'ensemble des condamnés, concessionnaires ou non, libérés à la date marquant la fin de leur peine. Ceux qui sont astreints à résidence sont désormais inscrits comme "libérés deuxième section». Ceux qui ne sont plus astreints à résidence sont inscrits comme «libérés première section». Cependant, le mot "libéré» renvoie en fait et communément à un groupe social inscrit dans les mémoires: des hommes ayant quitté le bagne sans concession, astreints à résidence, interdits de séjour à Nouméa sauf engagement de travail, qui nomadisent sur les chemins de la colonie, au gré des emplois éphémères qu'ils peuvent trouver dans les mines ou sur les propriétés pour survivre. 


\section{DOCUMENTS 3 et 4}

Le territoire de Koniambo

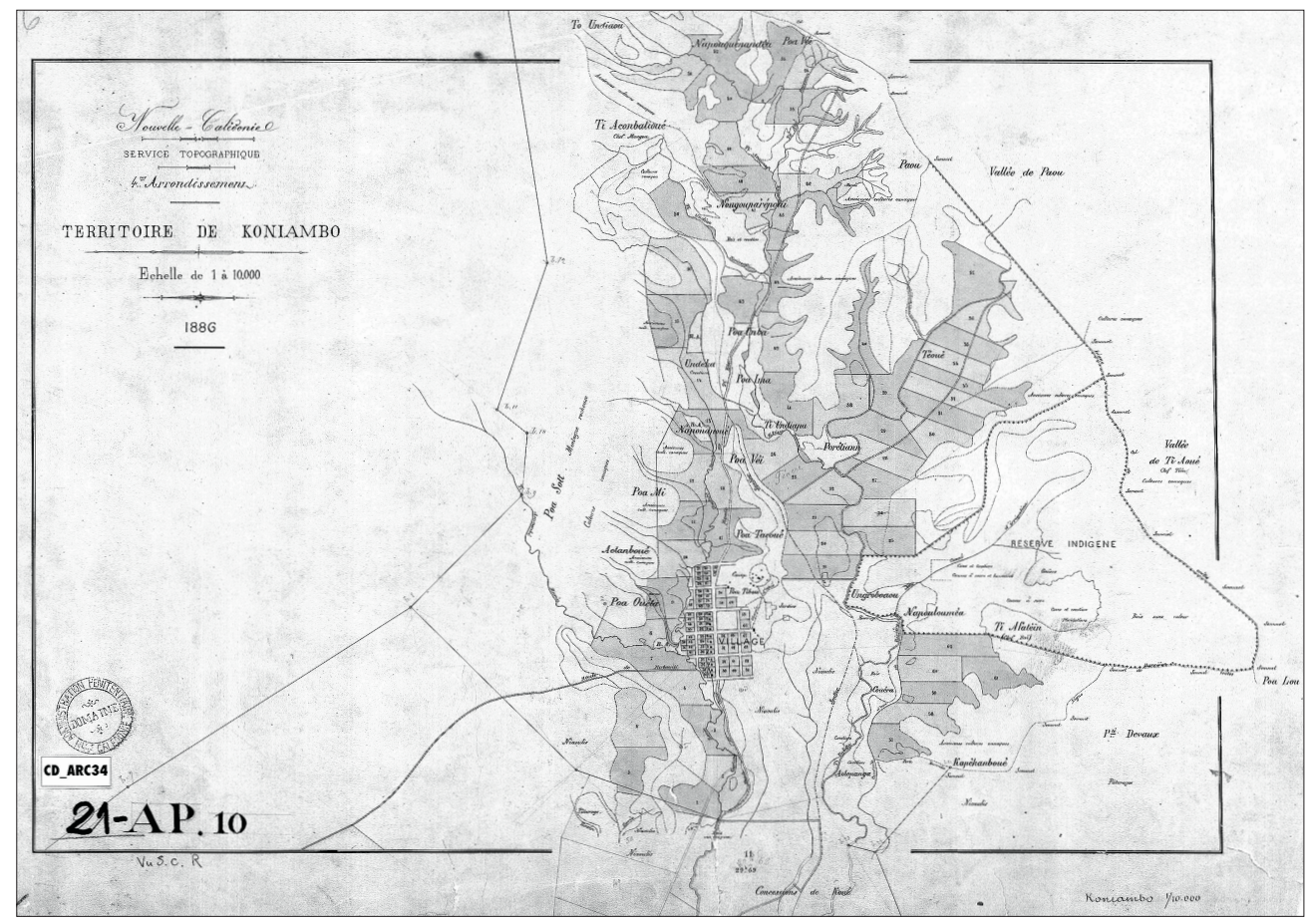

Source, Carte de Koniambo, 1886. Archives de Nouvelle-Calédonie, 21AP10.

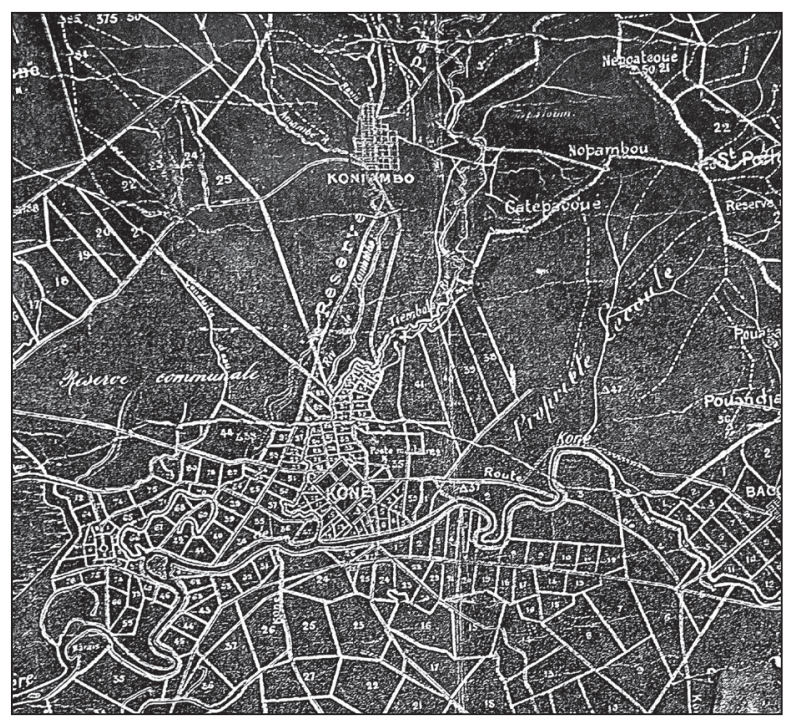

Source: carte de Koné/Koniambo, non datée (années 1900), archives municipales de Koné. 
les plus pénibles de la colonisation». Une fois l'épreuve passée, les hommes progressent de la $3^{\mathrm{e}}$ classe vers la $2^{\mathrm{e}}$ classe puis la $1^{\text {re }}$ classe, et leur condition s'améliore par étapes. En $1^{\text {re }}$ classe, une toute petite minorité (10\% seulement de l'effectif global des condamnés) se voit offrir la récompense suprême pour les concepteurs de la loi de 1854 sur la transportation: l'accès à la terre par l'installation sur concession en centre pénal. C'est à cette fin que des villages de concessionnaires pénaux sont ouverts, à Bourail en 1864, à La Foa/Fonwhary en 1871, à Pouembout en 1883 et à Koniambo, extension de Pouembout, en 1884.

Koniambo n'est situé qu'à trois kilomètres de Koné, centre de colonisation libre créé en 1880 tandis que Pouembout est éloigné d'une dizaine de kilomètres au sud. Ce petit centre de concessionnaires pénaux est donc inscrit dans un paysage dense, proche de colons libres - villageois de Koné ou éleveurs qui prennent de vastes propriétés dans la basse vallée ou les contreforts -, proche aussi de la réserve indigène de Koniambo et pas très éloigné des villages kanak qui se maintiennent plus loin dans les vallées de la chaîne.

On sait très peu de chose sur l'histoire de ce centre ${ }^{21}$. Lors de sa création en 1884, le condamné Daufelt raconte qu'une soixantaine d'élèves concessionnaires ont d'abord été logés dans des "cases canaques abandonnées" ou plus exactement que les habitants ont dû quitter dans des circonstances que l'on ignore. En sept mois, «un village modèle» selon lui est érigé. Un an plus tard, le missionnaire Rigard confirme: Koniambo «centre de concessionnaires en formation au sein d'une vallée qui touche au massif de la chaîne centrale [...] sera une ville ou tout au moins un bourg avec maisons groupées, rues alignées, places publiques». Mais il précise que "c'est un essai et qu'habituellement le concessionnaire habite sa concession - il vit isolé $»^{22}$. Un autre missionnaire décrit, en 1891, des concessions "très éparpillées sur un parcours de 5 kilomètres et dans le voisinage immédiat d'un village indigène assez important et de plusieurs mines $»^{23}$. Le centre végète, 12 concessions en 1884 , 18 en 1895 et 29 en 1910. Les terres y sont apparemment moins fertiles qu'à Pouembout, le centre pénal principal à une dizaine de kilomètres au sud. L'administration pénitentiaire abandonne progressivement le lieu, n'y installant à partir de 1912 les condamnés qu'au compte-gouttes ${ }^{24}$.

21. Quelques éléments trouvés dans le témoignage de missionnaires ou du condamné Daufelt (de son vrai nom Delfaut) écrit en 1895, un registre des concessionnaires établi à la même date suite à une enquête de l'administration pénitentiaire sur les centres de concessionnaires pénaux dans la colonie, quelques chiffres donnés par la Notice de la transportation: DAUfelt, Les damnés du Pacifique, Nouméa, Kiwanis Club, 1974; ANOM, FM, H/831, enquête auprès des concessionnaires pénaux de Pouembout, 1895; Notice sur la transportation à la Guyane française et à la Nouvelle-Calédonie... [titre suivi de l'année ou des années concernées], Paris, Imprimerie impériale puis Imprimerie nationale, 1852-1912.

22. Archives de l'archevêché de Nouméa (désormais AAN), 45.2, Rigard à Monseigneur, Fonwhari, le 27 juin 1885 .

23. AAN, 45.2, Chaboissier à Monseigneur, Koné, le 3 juin 1891.

24. En 1927, le curé de Koné déclare que «ce village n’a jamais connu la prospérité de celui de Pouembout, et c'est à peine s'il abrite actuellement une demi-douzaine de familles": AAN, 45.1, X. Chaboissier, "La mission de Koné de mai 1891 à janvier 1927». 
Les réglementations émises dans les années 1890 en Nouvelle-Calédonie, soucieuses de maintenir la ségrégation entre les catégories "libre» et "pénale» de la population, cherchent à limiter les contacts entre Kanak et libérés (interdisant à ces derniers de résider sur les réserves kanak, pénalisant le commerce d'alcool qu'ils peuvent faire dans les tribus et condamnant les relations qu'ils peuvent avoir avec les femmes kanak). Mais on peut douter de leur efficacité pratique dans des contextes locaux de forte proximité, comme c'est le cas à Koniambo où les terres occupées par les Kanak (la réserve de Koniambo et les terres de Grombaou) jouxtent les concessions pénales ${ }^{25}$. Les registres du tribunal de Koné font référence aux contacts entre libérés et Kanak de «Koniambo" et au commerce de l'alcool qui se développe ${ }^{26}$. La naissance d'enfants métissés témoigne de relations éphémères ou parfois durables. Mais plus que les libérés qui nomadisent sur les chemins de la colonie en quête de moyens de subsistance, les concessionnaires pénaux sont les voisins les plus stables et les plus proches des Kanak, convoitant souvent leurs terres.

C'est le cas de Claude Fiacre et de sa famille, en particulier son plus jeune fils Jean-Marie qui «serait bien aisé de s'arrondir au détriment des indigènes [...] moyennant une somme dérisoire", comme en témoigne un missionnaire en $1910^{27}$. Le père, originaire de l'Allier, a été condamné en 1879 à l'âge de 49 ans, pour meurtre, à la peine des travaux forcés à perpétuité. Il est alors marié à Rose Crouzier et père de six enfants dont deux mineurs. Il obtient, le 12 juillet 1885, deux lots ruraux (23 et 24) et un lot urbain (9) au centre pénal de Koniambo où il peut s'installer avec sa femme, ses deux plus jeunes enfants et deux de ses filles mariées, l'une à Jean Mercier et l'autre à Hippolyte Marin. Pour entretenir sa nombreuse famille, Fiacre se voit attribuer, en 1887 , deux lots ruraux $(64,65)$ et deux lots urbains supplémentaires $(56,64)$ où s'installent les couples Mercier et Marin. Cette belle extension est menacée en 1898 lorsque le gouverneur Feillet décide de déposséder les gendres «libres» abusivement installés, selon lui, sur des concessions pénales ${ }^{28}$, pour renforcer le caractère exclusif du peuplement pénal sur les territoires dédiés à l'administration pénale. Mais Hippolyte Marin engage des réclamations qui remontent jusqu'au ministre via son beau-frère resté en France ${ }^{29}$ et l'héritage est finalement transmis directement aux deux couples et au plus jeune

25. Il faut distinguer la réserve de Koniambo proprement dite sur laquelle se trouvent les terres de Noël à Tiamou, et qui recouvre 370 hectares, de Grombaou (ou Grombaoute/Gorobwau) couvrant 136 hectares qui avaient appartenu à l'administration pénitentiaire puis revinrent au Domaine pour être finalement transformés en réserve indigène en 1917.

26. A. MUCKLE, "Natives", "immigrants" and "libérés": the colonial regulation of mobility in New Caledonia", Law Text Culture, 15, 2011, p. 135-161, p. 146-147.

27. AAN, 45.1, "Questionnaire» [réponses probablement par de Thuret, Koné, ca. 1910].

28. ANOM, FM, H/691, dossier de transportation de Claude Fiacre; ANOM, FM, H/831, dossier de concessionnaire. Enquête de 1895.

29. ANOM, FM, H/691, dossier de transportation de Claude Fiacre. 
Jean-Marie à la mort du père en 1908. Jean-Marie Fiacre dispute de surcroît à ses voisins kanak, depuis 1909, les terres de Grombaou ${ }^{30}$.

Le chef Noël, quant à lui, est sous la pression directe de Joseph Gros, fils de Samuel Gros, condamné en 1874 à l'âge de 31 ans, pour meurtre, aux travaux forcés à perpétuité. Samuel Gros est déjà veuf lors de sa condamnation mais il a quatre enfants dont deux, Louis et Joseph, le rejoignent en Nouvelle-Calédonie ${ }^{31}$. Samuel est placé en 1885 sur le lot 4 de Koniambo. Jugé excellent concessionnaire, sa peine est commuée à 20 ans en 1886 et réduite progressivement par la suite. Il est autorisé à louer le lot 70 bis de Koniambo en 1899 et occupe en 1900, moyennant de faibles rétributions, "l'immeuble anciennement réservé à la cambuse" formant le lot 71 de Koniambo ainsi que les lots 1,7 et 10 soit quelque 258 hectares sur l'espace des concessions pénales $^{32}$. Cela ne suffit pas à son fils Joseph. Celui-ci cherche, en 1908, à s'emparer des terres de Noël à Tiamou, provoquant la plainte de ce dernier auprès du missionnaire en poste à Koné qui dénonce "un véritable déni de justice que l'on ne devrait pas laisser passer", les terres de réserve étant en principe inaliénables ${ }^{33}$ et légalement protégées de l'empiétement des colons. Mais Joseph Gros parvient à obtenir "par un marché de surprise " ${ }^{34}$ l'accord du petit chef Doui, qu'il enivre, pour s'emparer des terres de Noël sur lesquelles Doui n'a aucun droit. Se retrouvant très à l'étroit sur ce qui lui reste, Noël doit se résoudre à partir avec les siens pour s'installer plus loin, à Pwënaki sur la réserve de Goyeta-Pana. Dans une étude qu'elle consacre à la région, l'anthropologue Sonia Grochain insiste sur l'importance des agissements de Joseph Gros à l'encontre du chef Noël comme l'une des raisons principales du combat que ce dernier et ses alliés vont lancer en $1917^{35}$.

On notera que Claude Fiacre et Samuel Gros ont des profils similaires incarnant la "réussite» aux yeux de l'administration pénale: ils sont dotés d'une famille grâce à la venue de l'épouse et/ou des fils et filles. On sait toute l'importance du regroupement familial dans le soutien apporté au condamné concessionnaire pour favoriser son enracinement, surtout lorsque les fils ou

30. En juin 1910, le service du Domaine informe les habitants de Grombaou qu'ils occupent illégalement ces 136 hectares sur lesquels ils ont fait leurs champs et planté leur café. Ces terres sont convoitées par Jean-Marie Fiacre et Jules Azémat. Le Domaine demande alors aux Kanak de louer les terres pour 200 francs annuels ou de les abandonner. Ils devront dans ce cas payer rétrospectivement la rente annuelle due selon l'administration au titre de "l'occupation illégale»: AAN, 45.5, "Copie d'une lettre dont l'original est chez Doui chef de Koniambo: le chef du Service du Domaine aux indigènes Ch. Oué - Doui -J. Pounou - F. Mou etc. à Koniambo, signé Coulombeix, nº 761, Nouméa, le 13 juin $1910 »$.

31. Louis Gros, né en 1869, est arrivé à Koniambo en 1889 et il décède en 1904. Joseph est né en 1874 et a probablement rejoint son père avec son frère en 1889 à l'âge de 15 ans. On ignore la date de son décès ainsi que celle de son père.

32. ANOM, FM, H/610, dossier de transportation de Samuel Gros.

33. AAN, 45.4, Thuret à Monseigneur, Koné, le 16 mars 1908.

34. Ibidem.

35. Sonia Grochain, "Les Kanak et le travail en province Nord de la Nouvelle-Calédonie», thèse EHESS, 2007, p. 147-151. 
gendres déjà jeunes adultes peuvent aider aux développements commerciaux ou agricoles du père ou beau-père ${ }^{36}$. $\mathrm{Si}$, au moment de leur condamnation, Claude Fiacre est tailleur de métier et Samuel Gros marchand de vin, tous deux font partie de la population rurale française dont les origines paysannes sont proches. Loin d'être dépaysés, comme beaucoup d'autres condamnés souvent célibataires et parfois totalement ignorants du travail de la terre, Fiacre et Gros trouvent à Koniambo un univers accueillant dans lequel ils sauront se déployer. D'autant que l'un et l'autre sont dotés de ressources financières obtenues par la vente de biens en France alors qu'ils sont déjà en Nouvelle-Calédonie, et qu'ils peuvent réinvestir. Claude Fiacre parvient en 1900 à vendre au moins une maison et un immeuble à Coulanges dans l'Allier pour une valeur totale d'environ 4000 francs $^{37}$ tandis qu'un notaire adresse une lettre au ministre en 1876 pour retrouver Samuel Gros et "poursuivre la vente d'un usufruit" dans l'intérêt de ses enfants. On ne s'étonnera pas que les fils et gendres se montrent particulièrement doués pour faire fructifier l'héritage des pères, jouant de leur condition «libre» pour revendiquer auprès de l'administration la location de terrains sur les espaces pénaux ou supposément dédiés aux Kanak ${ }^{38}$.

Hippolyte Marin, on l'a vu, a su remonter jusqu'au ministre pour contrer en 1898 le gouverneur Feillet qui tente de le déposséder. Jean-Marie Fiacre hérite à la mort de son père en 1908 des lots urbains 23 et 29 (avec le titre définitif en 1912), cherche, en 1909-1910, à acquérir les 136 hectares de Grombaou et obtient en 1911 la location du lot rural $35^{39}$. Comme les fils Gros, il profite du déclin du bagne et de la désaffectation progressive du domaine foncier attribué à l'administration pénitentiaire pour consolider l'héritage acquis par le père grâce, si l'on peut dire, à sa condamnation. En 1916, Jean-Marie Fiacre, fils de condamné mais de condition libre, fait partie des notables du pays comme le prouve l'hôtel qu'il ouvre (dit hôtel Fiacre) à Koné $^{40}$. Louis Gros, quant à lui, a pu investir dans des concessions de terres à Koné. Il est propriétaire d'une station d'élevage à Oundja près de Panéqui. À sa mort, en 1904, il est noté comme «négociant» et célibataire. Son frère Joseph, outre les terres dont il hérite à Koniambo, est en 1918 propriétaire de trois stations d'élevage avec plus de 2000 hectares $^{41}$. Les uns et les autres,

36. I. MERLE, Expériences..., op. cit., p. 173-181.

37. Ce qui est une somme importante pour l'époque. À titre de comparaison, le capital de rachat des concessions pénales rurales de cinq hectares environ que doivent payer les condamnés lorsqu'ils obtiennent le titre définitif est de 2300 francs environ.

38. ANOM, FM, H/691 et H/610, dossiers de transportation de Claude Fiacre et Samuel Gros.

39. ANC, Conseil privé, séances du 28-29 juillet 1911 et du 2 mars 1912. Les lots de Claude Fiacre sont répartis entre ses trois enfants et gendres.

40. Brigadier FAURE, “"Affaires de Koné”...», art. cit., p. 79-80. En avril 1917, c'est à «l'hôtel Fiacre» que le commandant du «Kersaint» et le chef du service des Affaires indigènes se sont installés pour mener leur enquête sur les troubles dans la région.

41. AAN, 21.2, procès-verbal - audition de huit témoins [y compris Joseph Gros], $\mathrm{n}^{\circ} 349$, le 6 juillet 1918. 
quoiqu'en principe stigmatisés par leur ascendance pénale, ont su s'affranchir de la pesanteur des catégories coloniales imposées.

Dans le même temps, les Kanak de la région se trouvent dans une situation des plus vulnérable et s'appauvrissent grandement. Noël et les siens sont obligés de quitter Tiamou, lieu de repli pour la génération précédente suite à l'insurrection de 1878. En 1910, en raison de la pression exercée par JeanMarie Fiacre, les Kanak résidant à Grombaou se voient sommés de payer en location les terres qu'ils occupent et qui sont pour eux aussi des terres de repli car la plupart sont originaires des alentours du massif du Koniambo d'où ils ont été refoulés dans les années $1880^{42}$. Cantonnés sur les terres de réserves mais non moins menacés par la convoitise des colons libres et pénaux, les Kanak multiplient les stratégies de survie, tentant de se défendre en cherchant recours auprès des missionnaires mais parfois en remontant au gouverneur comme c'est le cas de Noël et des résidants de Grombaou. Obligés de payer l'impôt de capitation à partir de 1900 et de répondre aux réquisitions de l'administration, ils cherchent du travail auprès des colons ou sur les stations d'élevage comme c'est le cas pour Wâii, frère de Noël, qui travaille chez l'éleveur Metzdorf, ou pour Noël lui-même qui viendra travailler chez Mohamed ben Ahmed en 1916. Le missionnaire Niel en poste à Koné en 1910 écrit: «Sans le travail que font chez les blancs les indigènes des environs de Koné, ils seraient tous condamnés à mourir de faim»" ${ }^{43}$.

C'est dans ce contexte que Mohamed ben Ahmed est installé en 1916 sur le lot rural 34 à Koniambo. Il s'agit d'un lot ancien, occupé dès 1885 par le condamné français François Hervillard qui obtient sa libération en 1890 puis sa concession définitive en 1891 et meurt sans héritier en 1894. On ne sait quels ont été les occupants suivants si ce n'est le passage éphémère de Mohamed ben Ahmed, entre janvier 1916 et janvier 1918. Celui-ci reste très peu de temps car il doit fuir rapidement la vengeance des Kanak suite au meurtre du chef Noël.

\section{Un destin « ARAbe » entre Algérie et Nouvelle-Calédonie : Mohamed ben Ahmed}

Qui est Mohamed ben Ahmed et que sait-on de son origine et de son parcours?

Rappelons tout d'abord qu'il fait partie des 1822 indigènes d'Afrique du Nord transportés à la Nouvelle-Calédonie entre 1864 et 1897 et dont une large majorité arrive après 1889 , lorsque la décision est prise de cesser d'orienter les condamnés «arabes» vers la Guyane dont on prétend qu'ils s'évadent trop 
facilement pour les envoyer en Nouvelle-Calédonie ${ }^{44}$. Les concessionnaires «arabes» seront nombreux à être installés non loin de Bourail au lieu-dit Nessadiou, seul lieu où leur mémoire a laissé des traces, avec le cimetière musulman en bordure de route ou "le col des Arabes» à proximité, dont le nom rappelle leur présence ${ }^{45}$. Mais les concessionnaires «arabes» sont aussi éparpillés dans les autres centres pénaux, la Foa/Fonwhary/Farino et Pouembout, et les libérés "arabes" nomadisent comme les autres sur les routes de la colonie.

Les lettres des commissions municipales et les articles de journaux témoignent de cette présence «arabe» pour surtout dénoncer son caractère dangereux ou criminogène. On fustige «l'envahissement, les déprédations, les crimes des arabes", leur installation "plus ou moins licite», "la multitude de coreligionnaires" qu'ils attirent et même l'invasion de chèvres et autres bestiaux qui les accompagnent ${ }^{46}$. Les "Arabes" dans ce contexte sont particulièrement visés, victimes d'un racisme spécifique, victimes aussi des solidarités communautaires qu'ils savent entretenir bien plus que les autres transportés ${ }^{47}$.

Lorsque Mohamed ben Ahmed s'installe à Koniambo, il a 45 ans et une longue carrière de bagne derrière lui. Il y a déjà vingt-deux ans qu'il est en Nouvelle-Calédonie depuis son arrivée à l'île Nou en 1894.

La décision de son envoi en Nouvelle-Calédonie est prise par la commission de classement des condamnés aux travaux forcés, le 21 août 1894, sur la base d'un commentaire lapidaire: "que des mauvais renseignements sont fournis sur le compte du condamné» ${ }^{48}$. Comme le montre son dossier de transportation, nonobstant les confusions qui s'y glissent, Mohamed ben Ahmed a été condamné le 28 février 1889 par la cour d'assises d'Oran. Il a alors 20 ans semble-t-il. Il est le fils de feu Ahmed et de Zohra bent Moussa, enfant légitime né en 1869, à Beni Snassen, ce qui en fait un ressortissant du Maroc, originaire des zones frontalières entre Maroc et Algérie à proximité d'Oran. Il est précisé qu'il est marié en 1889 mais le nom de l'épouse reste inconnu. De religion musulmane, il ne sait ni lire ni écrire, appartient à la population rurale et se définit comme «journalier». La notice individuelle indique qu'il travaillait pour autrui mais qu'en fait il n'exerçait pas réellement sa profession et vivait dans l'oisiveté tout en étant parfaitement apte au travail. À la question "Comment était-il noté dans sa commune?», le greffier écrit "mal». Le fait est qu'il fut arrêté après avoir attaqué, avec huit autres

44. On consultera utilement sur ce point la thèse récente de Marine COQUET, «La ville et le bagne. Histoire d'une commune pénitentiaire en terre coloniale, Saint-Laurent-du-Maroni, Guyane, 1857-1949", thèse EHESS, 2016.

45. À propos des Maghrébins transportés en Nouvelle-Calédonie: Mélica OUENNOUGHI, Algériens et maghrébins en Nouvelle-Calédonie. Anthropologie historique de la communauté arabo-berbère de 1864 à nos jours, Alger, Casbah Éditions, 2008.

46. Commission municipale de Voh, 12 février 1903.

47. I. MERLE, Expériences..., op. cit., p. 368-371.

48. ANOM, FM, H/1 557, commission de classement aux travaux forcés, dossier de transportation, Mohamed ben Ahmed. 
"marocains", des villageois de Djemaa Sacra se rendant de nuit au marché de Tlemcen, le 4 avril $1888^{49}$.

Mais Mohamed s'évade, alors qu'on le transfère d'Oran à Alger, en sautant du train en marche, et gagne la frontière du Maroc pour se cacher. Il reste en fuite pendant quatre à cinq ans au cours desquels il a un enfant. Son erreur fut de repasser la frontière algéro-marocaine le 2 mars 1894 pour aller visiter un marabout à la Zaouiia d'Atkia. C'est là qu'il est arrêté par les gens de la tribu et remis entre les mains de l'autorité militaire ${ }^{50}$.

C'est donc en 1894 qu'est prise la décision de l'expédier en Nouvelle-Calédonie afin qu'il purge la peine contractée en 1889. Mohamed est embarqué sur Le Calédonie le 30 octobre et débarqué à Nouméa le 17 décembre 1894. Il fait partie des 287 "condamnés arabes» débarqués cette année-là à Nouméa, chiffre annuel le plus élevé de transportés «arabes" arrivant sur l'île pendant toute la période de la transportation entre 1864 et 1897.

Une fois Mohamed ben Ahmed en Nouvelle-Calédonie, des temps très difficiles débutent pour lui. Il n'est pas un condamné modèle. Il tente une première évasion le 7 mars 1895, à peine un an après son arrivée en NouvelleCalédonie, mais il est réintégré dès le 17 juillet 1895 pour être condamné par le tribunal maritime spécial à deux ans de double chaîne. Il retente sa chance le 2 juin 1898 mais est repris le 21 août 1898 et condamné par le même tribunal à la même peine. Il est à nouveau condamné le 20 mai 1902 pour évasion dont la durée couvre cette fois presque un an (31 mars 1901-8 février 1902), aggravée par un vol qualifié, ce qui le conduit à purger une peine de quatre ans de réclusion cellulaire. Ces évasions successives témoignent à la fois de l'irrépressible envie de fuir qui touche nombre de condamnés mais aussi de l'extrême difficulté de l'aventure dans une île où il n'existe pas de lieu refuge sûr, où les Kanak reçoivent des primes pour rattraper les condamnés, et où la présence du personnel de bagne surveillant les chantiers de routes, celle des contremaîtres sur les mines éparpillées et celle des gendarmes, des colons et des éleveurs, engendrent un risque constant de dénonciation.

C'est au cours de sa période de prison que Mohamed fait une première demande de grâce qui transite lentement entre 1903 et 1905 vers le ministère de la Justice puis le ministère des Colonies pour redescendre vers le gouverneur. Il obtient le 15 février 1905 une remise de sa peine réclusionnaire mais voit son recours en grâce rejeté. Sont alors énumérées les punitions qu'il a subies depuis 1894, outre les condamnations pour évasions: 2 jours de pain sec pour paresse au travail, 22 nuits de prison pour mensonge (non malade) et infractions, 37 jours de salle de discipline pour bavardage, non malade, rixe et infraction, 15 jours de cellule pour trafics d'effets, 30 jours de cachot pour paresse et bavardage et enfin, en 1903, 3 jours de cachot pour bavardage.

49. Ibidem, exposé sommaire des faits qui ont motivé la condamnation à subir.

50. Ibidem, interrogatoire mené au tribunal de première instance de Tlemen, le 16 mars 1894. 
La conduite est jugée médiocre, plusieurs condamnations ont été prononcées depuis son arrivée dans la colonie dont deux pour vol (mais le dossier n'en indique qu'une). La conclusion tombe le 15 septembre 1905: «Ce condamné étant de $3^{\mathrm{e}}$ classe, ne réunit pas les conditions nécessaires pour une grâce " ${ }^{51}$.

Visiblement Mohamed s'assagit entre 1905 et 1916, trop découragé peutêtre pour tenter l'évasion, trop écrasé par le régime du bagne. On ne sait rien de cette période, ni le lieu où il travaille et réside, dans quel camp, chantier ou pénitencier.

Mais par sa bonne conduite il obtient que sa peine de travaux forcés à perpétuité soit commuée en peine de 20 ans en $1912^{52}$ et progresse dans la hiérarchie des condamnés, de la $3^{\mathrm{e}}$ classe jusqu'à la $1^{\text {re }}$. En 1916, lorsqu'il se voit proposer une concession, l'homme est certainement usé par les longues années de bagne qu'il a traversées mais, comme beaucoup de ses compagnons de peine, il est au mitan de sa vie et peut enfin goûter à la liberté, même si celleci reste conditionnelle, et au plaisir du travail de la terre pour ce cultivateur et fils de cultivateur de la région d'Oran sur son lot rural 34 de Koniambo. Sa rencontre avec Noël va en décider autrement.

\section{Au CROISEMENT DE DESTINS INDIGÈnES ET COLONIAUX: LES RAISONS D'UN MEURTRE ET LES CONSÉQUENCES}

Pourquoi Mohamed ben Ahmed en vient-il à tuer Noël et à le décapiter? Lui qui fut "un rebelle» dans le contexte du bagne en Nouvelle-Calédonie, cherchant à s'évader à plusieurs reprises, s'est-il finalement rallié à l'autorité coloniale, prêt à servir d'auxiliaire aux forces militaires françaises comme d'autres l'ont fait, y compris les Algériens transportés dans les années 1870 et venus soutenir les troupes françaises lors de l'insurrection kanak de 1878 ? On sait que la mémoire est persistante dans les villages kanak et, de fait, Noël - luimême issu d'une famille touchée par la répression de l'insurrection de 1878 et qui a dû fuir Poya - évoque devant Mohamed des souvenirs douloureux:

«Celui-ci lui aurait parlé de l'insurrection de 1878 en disant qu'à cette époque les arabes avaient tué beaucoup d'indigènes mais que si cela se renouvelait aujourd'hui les Canaques tueraient d'abord les arabes. Ces paroles lui étant revenues à la mémoire au moment où il préparait le café qu'il servit à Noël, c'est à ce moment qu'il décida de le tuer avant que lui ou ses partisans ne le tuent lui-même, comme ils avaient déjà tué d'autres colons " ${ }^{53}$.

Mohamed aurait donc agi par peur plutôt que par véritable ralliement à la cause coloniale, évoquant la crainte d'une vengeance au long cours liée au traumatisme de l'insurrection de 1878 pour les Kanak et au souvenir des

51. Ibidem, demande de recours en grâce.

52. Ibidem, garde des Sceaux au ministre des Colonies, 10 octobre 1921.

53. Rapport du tribunal maritime spécial, île Nou, 25 mai 1918, in Nicolas RATZEL, Cahiers de mes souvenirs de géomètre calédonien, 1894-1939, Nouméa, Société d'études historiques de la NouvelleCalédonie, 2006, vol. 2, p. 38-39. 
trahisons et collaborations de l'époque, en particulier celle des condamnés "arabes", enrôlés alors dans les battues organisées par les colonnes militaires françaises et particulièrement redoutés pour leurs aptitudes de cavaliers, de pisteurs et pour leur violence guerrière ${ }^{54}$. En cela, il n'est pas le seul car nombre de colons évoquent alors la peur des représailles kanak et redoutent les tensions liées à la mémoire des événements, comme en témoigne un article publié le 26 janvier 1918:

«Il a été remarqué les regards de colère et de haine que lançaient aux habitants, nos soi-disant alliés de Baco, Koniambo et autres tribus voisines, quand ils apprirent la mort de Noël. Il sera donc prudent de ne pas s'en remettre complètement à leurs protestations d'amitié et à leurs offres de service» ${ }^{55}$.

La peur des Kanak explique la fuite immédiate de Mohamed ben Ahmed de sa concession après son crime pour se réfugier à Pouembout. Mais il n'hésite pas non plus, dans une lettre qu'il écrit au gouverneur en 1918, à revendiquer son acte:

«Monsieur le Gouverneur, bien que je suit encore en cour de peine, je croit être méritant puisque j'ai rendu un grand service à la justice ainsi qua toute la population en ayen la vie de ce terrible canaque redouté de tout le monde. Je viens donc suplier Monsieur le Gouverneur de sa puissante intervantion pour avoir égard a mon faite care depuis quil est mort touts les autres se son rendus $\rangle^{56}$.

En fait, comme il l'avoue lui-même, il connaissait «parfaitement» Noël avant le déclenchement des hostilités en 1917 car il l'employait sur sa concession en 1916. On sait que les Kanak avaient pour obligation de répondre aux injonctions du syndic des affaires indigènes (le gendarme) pour aller travailler chez les colons libres pendant les récoltes. Certains sont poussés à s'engager plus longuement sur une année ou plus. Pour les colons pénaux, l'administration pénitentiaire exigeait plutôt que ces derniers travaillent eux-mêmes leur plantation. Mais à l'évidence, sur la base de relations interpersonnelles, certains (et c'est aussi vrai pour les colons libres) demandent directement aux Kanak de venir les aider et c'est le cas de Noël qui travaille chez Mohamed. Ce dernier argue qu'il n'aurait pu le tuer s'il ne l'avait pas bien connu car Noël, très méfiant, ne se serait pas laissé surprendre. Et sa dernière parole avant de mourir témoigne de cette confiance trahie qu'il accordait à Mohamed, en s'écriant «Ah! Salaud!».

Les Kanak témoignèrent eux aussi que Mohamed connaissait bien Noël. Son frère Wâii (Ouai) affirme devant le gendarme Médoux en 1918: «Je sais qu'avant l'insurrection indigène mon frère Noël allait voir presque tous les

54. C'est ce qu'affirmait J. Delathière lors d'une conférence donnée devant des étudiants de l'université de Nouvelle-Calédonie en 2013 à La Foa. Jerry DELATHIĖRE, "CiRi, le terroir assassiné. Impact du fait colonial sur l'organisation foncière et sociale d'un terroir kanak d'origine", mémoire de diplôme de l'EHESS, 2006.

55. Le Bulletin du Commerce, 26 janvier 1918.

56. ANC, 1W1, lettre de Mohamed ben Ahmed adressée au gouverneur le 18 mars 1918. 
jours l'arabe Mohamed chez lui ${ }^{57}$. Liguori, Kanak pro-français de Koniambo, affirme que selon la femme de Noël, Dina, celui-ci s'était rendu chez Mohamed «7 jours» avant sa mort. Il ajoute:

«Avant l'insurrection indigène, l'arabe passait à cheval à la tribu tous les 2 ou 3 jours, pour chercher des vivres à Koné et en revenait avec un petit sac. Mais depuis l'insurrection, il allait aux vivres à peu près tous les 2 ou 3 jours mais en revenait avec un sac rempli et très volumineux attaché sur sa selle. Les indigènes de Koniambo se sont rendus compte également de cela et ont pensé comme moi que la plus grande partie de ces vivres était destinée à Noël. En outre, depuis ce moment, l'arabe ne demandait plus aucun indigène pour travailler chez lui comme auparavant ${ }^{58}$.

C'est justement cet approvisionnement qui semble grossir pendant l'insurrection qui fait porter le soupçon sur Mohamed et le soutien éventuel qu'il a apporté aux Kanak. Wâii, le frère de Noël, affirme:

"Quelques jours après l'affaire de Tiamou [...] j'ai vu mon frère Noël avec un pain et cinq douilles vides pour calibre 12 millimètres. Lui ayant demandé la provenance de cet objet, Noël m'a dit que c'était l'arabe Mohamed de Koniambo qui les lui avait donnés " ${ }^{59}$.

Et Liguori accuse Mohamed d'avoir tué Noël en confiance alors que celui-ci allait se rendre aux autorités à Koné. Wâii ajoute qu'il était porteur d'un drapeau blanc ${ }^{60}$.

Mohamed nie avoir fourni des vivres mais le gendarme Médoux précise: «Dans les premiers jours de juin 1917, des soupçons planaient déjà sur l'arabe Mohamed et son voisin, le condamné Prosi [Prési], actuellement concessionnaire à Pouembout ${ }^{61}$. Il poursuit en déclarant qu'une enquête a été ouverte avec les interrogatoires des colons Hervouët et Azémat chez qui Mohamed et Prési venaient se ravitailler.

Des observations ultérieures déplacent cependant les soupçons sur les agissements de colons, comme en témoigne un rapport de l'adjudant-chef Durand relatant la déposition qu'il a reçue de l'indigène Téin de la tribu de Pana, et résumé dans une lettre adressée par le gouverneur au procureur général le 29 mai 1918:

«Il résulte de ces déclarations de ce rebelle que trois commerçants, les sieurs Fiacre, Azémat et Magnier auraient vendu aux insurgés, antérieurement à la rébellion, mais depuis le début des hostilités en Europe, un certain nombre de fusils, ainsi que de la poudre de chasse. D'autre part, l'un des trois frères Metzdorf, propriétaires éleveurs à Voh aurait tenu

57. ANC, 1W1, Médoux, procés-verbal, Koné, 8 mars 1918.

58. Ibidem.

59. Ibidem

60. Ibidem

61. Ibidem. Il s'agit de Louis Prési, sujet italien, condamné une première fois en Corse pour homicide volontaire, le 20 novembre 1890, à dix ans de travaux forcés et à la transportation. Prési est libéré en 1900 et fait plusieurs recours pour obtenir la remise de résidence obligatoire mais se fait à nouveau prendre dans une rixe et est condamné pour homicide à dix ans de travaux forcés le 5 octobre 1909. Il obtient une concession de terre à Pouembout en 1916 et sa libération en 1919. ANOM, FM, H/1 493, dossier de transportation de Louis Prési. 
au chef rebelle Noël, au moment des opérations de recrutement des volontaires indigènes pour la durée de la guerre, des propos de nature à nuire à l'enrôlement des tirailleurs ${ }^{62}$.

Le 8 février précédent, lors d'un interrogatoire, Poindet Apengou signalait que les habitants d'Atéou, Pamalé, Ouamindiou et Néami écoulaient de préférence leurs produits chez François Hervouët, Antoine Chautard et Jean-Marie Fiacre qui, en échange de café, leur fournissaient des fusils et de la poudre ou même des cartouches ${ }^{63}$.

Les noms cités ici sont très connus dans le pays de Koné/Koniambo. On l'a vu avec Jean-Marie Fiacre, fils de concessionnaire pénal qui tient alors un hôtel, ou Joseph Gros qui possède alors trois stations et plus de 2000 hectares. Antoine Chautard est fils d'un colon du même nom installé à Koné en $1891^{64}$. Jules Azémat ${ }^{65}$, originaire de Seine-et-Oise, arrivé comme colon à Koné en 1899, François Hervouët ${ }^{66}$, frère d'Alexandre qui s'installe à Koné dans les années 1895 ou encore Joseph Magnier sont, quant à eux, tous d'origine libre, colons et propriétaires fonciers. Certains sont détenteurs d'un store (magasin), en particulier Joseph Magnier dont la maison de commerce reprise par son fils Maurice deviendra l'une des plus importantes du village après la guerre de 14-18. Les trois frères Metzdorf sont originaires de Belgique et éleveurs, possédant de grandes propriétés dans la chaîne ${ }^{67}$.

On devine ainsi, à travers les indices donnés par les différentes enquêtes, une collusion d'intérêts entre ces colons et commerçants enrichis, les condamnés concessionnaires beaucoup plus modestes comme Mohamed ben Ahmed et Prési, et les Kanak insurgés. Les motivations des uns et des autres et la nature des relations que ces individus entretiennent restent obscures, mais on peut tenter quelques hypothèses à partir des traces laissées dans les archives.

On peut supposer que les commerçants de Koné, tels qu'Azémat ou Magnier ou encore Fiacre et Hervouët, voient un intérêt direct à troquer le café récolté par les Kanak contre les fusils, la poudre et les cartouches que ces derniers demandent, s'assurant ainsi une clientèle et des revenus. Les

62. ANC, 1W1, Repiquet au procureur général, 29 mai 1918. Le rapport de l'adjudant Durand du 27 avril 1918 n'a pu être retrouvé.

63. Ibidem; AAN, 21.1, interrogatoire de Poindet, ex petit chef du village de Paola-Netchaot, la Prison Civile, 8 février 1918.

64. Antoine Chautard (1837-1912), installé à Koné en 1891, a trois fils dont le dernier s'appelle aussi Antoine.

65. Jules Azémat (1872-?1931), fils d’Émile, arrivé en 1899 à Koné, originaire de Corbeil en Seine-et-Oise. Identifié en 1918 comme "propriétaire et commerçant à Koné ", il est marié à Juliette Monnier. Jules a employé, avant le 28 avril 1917, deux frères de Noël, Poigny et Ouagni [Wâii] de Tiamou, pendant trois mois sur sa propriété de Poindio. AAN, 21.2, procès-verbal - audition de huit témoins [y compris Jules Azémat et Juliette Monnier dame Azémat], nº 349, le 6 juillet 1918; Bulletin du Commerce, 12 mai 1917.

66. François Hervouët était le fils d'Auguste Hervouët, famille de colons Feillet arrivée de Vendée, installée à Koné en 1893 avec ses cinq enfants.

67. Charles Marie Félix Metzdorf est né en 1843 à Ypres en Belgique et meurt à Koné en 1884. On sait qu'il a eu trois fils nés à Bruges, Charles Jacques (né en 1867), Alfred (né en 1869) et Edmond qui restera avec une femme kanak et s'installera à Voh. 
frères Metzdorf, éleveurs qui utilisent les Kanak comme stockmen, semblent décourager le recrutement des volontaires indigènes pour la guerre. Le fontils pour garder une main-d'œuvre auprès d'eux? Ils ne seraient à l'évidence pas les seuls. Un communiqué publié dans La France Australe, le 15 février 1916, signale que des engagistes refusent à leurs engagés le droit de se porter volontaires dans l'armée sous prétexte qu'ils sont déjà sous contrat avec eux. Le communiqué doit rappeler que l'enrôlement militaire prévaut sur toute autre forme d'engagement ${ }^{68}$.

On doit aussi envisager l'intérêt que présente le développement d'une insurrection kanak dans le contexte de 1917: elle permettra de retenir les mobilisés sur place pour mener les opérations de répression plutôt qu'ils partent au front en France, dont les nouvelles sont alors très mauvaises. Les familles des soldats reçoivent des lettres éprouvantes ou, pire, des avis de décès ${ }^{69}$. Le retour des permissionnaires à partir de mars 1917 et les témoignages qu'ils apportent contribuent à rendre l'atmosphère pesante. La pression de la guerre est à son comble dans les centres de colonisation libre comme dans les tribus au cours de l'année 1916 et reprend de plus belle en février 1917, lorsque le gouverneur appelle à la mobilisation générale de tous les hommes en âge de porter les armes jusqu'à l'âge de 48 ans, comme en métropole, ainsi qu'au recrutement massif des "volontaires» kanak. Parmi les Européens, cependant, il est prévu de donner la priorité aux soldats de métier et aux classes les plus jeunes de 1916, 1917 et 1918 jusqu'à concurrence de 400 hommes. L'enjeu est de recruter 500 volontaires kanak pour remplir le navire et éviter d'accroître le nombre des mobilisés d'origine européenne. Les réticences, on le sait, sont très fortes dans nombre de tribus et la tension autour des questions de recrutement palpable. Au sein de la population européenne, alors que les permissionnaires manifestent pour ne pas repartir ${ }^{70}$, les colons libres plus âgés profitent des sursis qui leur sont accordés pour éviter tout risque de départ et d'enrôlement. Parmi la liste de sursitaires publiée dans La France Australe le 10 mars 1917, on trouve à Koné Jean-Marie Fiacre, Jules Azémat et Joseph Gros $^{71}$. Les deux premiers, soupçonnés plus tard d'avoir vendu des armes ou munitions aux Kanak, n'ont pas eu besoin de le faire pour empêcher leur propre départ vers le front mais l'idée est venue à d'autres. C'est le cas de François Hervouët qui aurait déclaré en 1917 à Koné : "C’est bien ennuyant d'être mobilisé et je donnerai bien 100 frs pour que les Canaques descendent de leurs montagnes nous empêchant de partir ${ }^{72}$.

68. La France Australe, 15 fevrier 1916.

69. Le premier convoi est parti en avril 1915 avec à son bord des mobilisés citoyens français. Les deux suivants, qui ont quitté l'île en juin et décembre 1916, comptaient des mobilisés et des volontaires kanak et tahitiens.

70. A. MUCKLE, Specters..., op. cit., p. 81.

71. La France Australe, 10 mars 1917. Au total, on trouve 145 sursitaires.

72. ANC, 1W2, Bouju (commandant du Kersaint) à Repiquet, $\mathrm{n}^{\circ} 196$, le 16 avril 1917; voir A. MUCKLE, Specters..., op. cit., p. 24-26. 
La suspicion existe comme en témoigne le gendarme de Pouembout, Rubod. Celui-ci affirme au procès des insurgés en 1919 qu'une manifestation des colons organisée le 5 avril 1917 à Koné «aurait été provoquée pour maintenir les mobilisés dans la colonie! ${ }^{73}$ Celle-ci eut lieu lorsque les parents et mobilisés, regroupés sur le wharf de Koné pour assister au départ de ces derniers, apprirent que quelques Kanak en armes s'étaient présentés au village. Selon une note rédigée en 1927 par l'archevêque Chanrion, feu Camille Caujolle (mort en mars 1918), propriétaire de la station d'élevage de Poindah, avait payé les «rebelles de la rivière pour qu'ils fassent la guerre ce qui devait lui éviter d'être mobilisé et peut-être envoyé en France ${ }^{74}$. Il faut rappeler ici que la mobilisation des colons d'origine libre alimente, depuis le début de la guerre, les plus vives rumeurs sur l'insécurité qu'elle peut provoquer en brousse du fait du départ des hommes et des possibles révoltes kanak qui en découleraient. Dans ce climat d'insécurité, il semble tout à fait possible que certains ne se contentent pas d'agiter les peurs mais fournissent des armes aux Kanak contre du café pour entretenir les troubles et retenir au maximum les hommes en âge de combattre au pays. Il existe, selon l'anthropologue Jean Guiart, auteur du premier article sur la guerre de 1917, des traditions familiales en Nouvelle-Calédonie pour évoquer ces stratégies ${ }^{75}$.

Peut-être aussi les colons de l'époque agissent-ils en connivence avec les Kanak car il est à noter que les colons dont on parle sont très insérés dans le tissu local. Antoine Chautard est interprète pour l'adjudant-chef Durand en 1918 et participe au procès des insurgés en 1919, même si les avocats de la défense affirment qu'il ne connaît qu'imparfaitement les "idiomes de la région de Koné» ${ }^{76}$. Jean-Marie Fiacre a été contremaître chez le colon Leconte dans sa jeunesse où il a donc été au contact de la main-d'œuvre kanak, néohébridaise ou javanaise ${ }^{77}$. En 1909, un gendarme déclare que Jean-Marie Fiacre et un métis qui l'accompagne, Dick Maccan, sont «depuis de longues années au courant des agissements des Canaques dans la région» et "causent bien la langue des indigènes et ceux-ci leur confient, volontiers, tout ce qu'ils savent ${ }^{78}$. En 1916, Fiacre est suffisamment lié avec certains Kanak pour proposer à Tein-René de Poamoa-Pana de lui louer les lots 23 et 29 de sa

73. La France Australe, 26 août 1919; voir A. BENSA, K.Y. Goromoedo, A. MUCKLE, Les sanglots..., op. cit., p. 142 .

74. AAN, 131.1, «Koné - faits de 1917-1919» et "Notes diverses sur Affaires concernant les tribus de Koné», signé Claude Marie Chanrion, Nouméa, le $1^{\text {er }}$ février 1927.

75. Thèse défendue par: Jean GUIART, «Les événements de 1917 en Nouvelle-Calédonie», fournal de la Société des Océanistes, 26-29, 1970, p. 265-282. Il dit avoir recueilli de la bouche de Monsieur Garrigou et de maître Vergès un récit entendu dans leurs familles respectives "attribuant l'origine de l'affaire de 1917 aux désirs des milieux de colonisation de la région de Koné et Voh voulant éviter le départ en France des jeunes classes mobilisées, qu'on devait d'ailleurs effectivement maintenir dans le territoire pour assurer l'ordre par suite des "événements" " (ibidem, p. 265).

76. La France Australe, 7 juillet 1919.

77. ANC, 23WE1, jugement de simple police, $\mathrm{n}^{\circ} 235$, le 13 novembre 1899.

78. ANOM, 38APC1, rapport n 184 de la brigade de Koné, le 16 août 1909, p. 61. 
propre concession pour 1200 francs par an; proposition que l'administration refuse car les Kanak pourraient ainsi résider hors des réserves et parce que Fiacre est connu pour son intempérance et sa brutalité envers ces derniers ${ }^{79}$.

Qu'en est-il finalement des condamnés tels que Mohamed ben Ahmed ou Prési, eux aussi accusés d'avoir soutenu l'insurrection? S'ils ne sont pas directement touchés par le recrutement de la guerre (parce qu'en cours de peine), ils ont certainement intérêt à gagner quelques sous auprès des commerçants de Koné et des Kanak en remontant le ravitaillement à la réserve. Leur situation de concessionnaire est bien plus précaire que celle des familles Fiacre ou Gros. Célibataires, installés depuis peu de temps sur leur lot, ils peinent sans aucun doute - comme beaucoup d'autres - pour se maintenir et l'argent frais, même en quantité négligeable, peut les avoir poussés à répondre à la demande kanak. On peut aussi imaginer qu'il s'agit d'une affaire d'interconnaissance et peut-être d'amitié, nouée en particulier entre Mohamed et Noël. Le premier choisit d'abord de donner asile et ravitaillement au second pendant les premiers mois de l'insurrection et l'accueille pour partager du café et du pain. Ces hommes ont travaillé ensemble, se connaissent assez bien semble-t-il et s'apprécient peut-être. On sait qu'à la veille de l'insurrection, le 27 avril 1917, un "Arabe» occupant une concession de Koniambo aurait reçu Noël et l'aurait prévenu qu'il risquait d'être attaqué par certaines personnes de Koniambo et de Tiaoué après la cérémonie organisée pour le lendemain ${ }^{80}$. Tout porte à croire qu'il s'agit de Mohamed ben Ahmed qui semble faire partie des personnes ayant prévenu Noël du guet-apens qui l'attendait. Mohamed voulait-il témoigner de son amitié ? Faut-il y voir des motivations plus politiques: le soutien à une révolte de "frères colonisés", une vengeance contre le système de colonisation qui, via la transportation, l'avait lui-même broyé ? On ne peut le savoir. Mais on sait que finalement, neuf mois plus tard, Mohamed assassine Noël.

Pourquoi? Peut-être par appât du gain: la prime de 2000 francs offerte pour la tête de Noël est une somme très importante pour l'époque, correspondant quasiment au prix de rachat d'une concession de cinq hectares. On ne peut ignorer l'attrait d'un tel montant pour un misérable concessionnaire comme Mohamed. Mais on peut aussi imaginer que celui-ci tue Noël sur un coup de tête lorsqu'il apprend par ce dernier qu'il descend à Koné pour se constituer prisonnier avec le risque qu'on l'interroge et qu'il évoque l'aide qu'il a reçue de «l'Arabe», le dénonçant par là même. La crainte de retourner à nouveau au bagne autant que l'opportunité de gagner la prime ont coûté la vie à Noël par la main de Mohamed.

79. ANOM, Affaires politiques 742, Pégourier (Inspecteur colonial), rapport du $1^{\text {er }}$ mai 1919.

80. ANOM, Affaires politiques 741 , procès-verbal d'interrogatoire des indigènes de la circonscription de Koné, Prison Civile, le 6 décembre 1917; voir A. BEnsA, K.Y. Goromoedo, A. MUCKLE, Les sanglots..., op. cit., p. 69. 


\section{DÉNOUEMENT}

Celui-ci, terrorisé à la suite de son acte par le risque qu'il court, à rester sur sa concession, de voir s'abattre la vengeance des Kanak, se réfugie chez son ami kabyle Areski ${ }^{81}$, lui aussi condamné installé à Pouembout, comme il le précise dans une lettre adressée au gouverneur le 18 mars $1918^{82}$. Un télégramme de la gendarmerie, le 5 avril 1918, note que Mohamed «ne peut pas trouver de travail à Pouembout parce qu'on suppose qu'il a lâchement assassiné le chef Noël au moment où il allait se rendre ${ }^{83}$. Le 3 mai 1918, le gouverneur de la Nouvelle-Calédonie demande que la prime de 2000 francs lui soit versée même si le procureur fait savoir en 1919 qu'il la trouve injustifiée ${ }^{84}$.

À cette date, d'ailleurs, tous les soupçons envers Mohamed et Ducamp ont été levés par le tribunal maritime spécial chargé de juger les condamnés et libérés, organisé en mai 1918 à Koné. Les accusations de collusion avec les insurgés sont alors oubliées et Mohamed est excusé du fait d'avoir osé porter une arme en dépit de sa condition pénale:

«Dès le commencement de la rébellion, tous les colons s’étaient armés pour défendre leur vie et leurs biens. Mohamed a trouvé l'occasion d'acheter un revolver pour sa défense personnelle sachant très bien que sa situation de condamné le lui défendait, mais en raison des circonstances, j'estime qu'il ne peut lui en être tenu rigueur. Mohamed a commis un homicide volontaire, c'est un fait certain et reconnu, mais s'il a commis cet homicide, c'est parce qu'il a cru sa vie en danger, et l'on ne peut prouver le contraire, surtout si l'on pense aux massacres commis par les rebelles; donc l'excuse de l'acte qu'il a commis doit être admise» ${ }^{85}$.

Le tribunal maritime spécial conclut à un non-lieu pour les deux hommes. Le 3 juillet 1918, l'administration pénitentiaire accorde à Mohamed ben Ahmed le lot 202 à Pouembout en remplacement du lot 34 de Koniambo pour le mettre à couvert. Son dossier de transportation nous indique qu'il fait un recours en grâce en 1921 et obtient une remise de peine de cinq ans ${ }^{86}$. En 1928 , il est libéré $4^{\mathrm{e}}$ catégorie $2^{\mathrm{e}}$ section sous le numéro 13753 , c'est-à-dire avec astreinte à la résidence perpétuelle ${ }^{87}$. Il fait un nouveau recours en grâce en 1931 pour lever la résidence perpétuelle mais la demande reste sans réponse

81. Il s'agit d'Arezki ben Moktar qui n'a pas de concession mais s'emploie comme gardien de bétail chez un colon à Pouembout depuis 1912 au moins, selon une lettre envoyée par le gouverneur au ministre des Colonies le 20 novembre 1912. Arezki ben Moktar, né en 1857 à Tizi Nterga (Tizi Ouzou), a été condamné en 1895 à quinze ans de travaux forcés pour assassinat. Il est en contact avec sa famille qui demande en 1913 la remise de sa résidence perpétuelle en Nouvelle-Calédonie, qu'il obtient le 22 novembre 1917: ANOM, FM, H/1 265, dossier de transportation d'Arezki ben Moktar.

82. ANOM, FM, H/1 567, dossier de condamné, Mohamed ben Ahmed.

83. ANC, 1W1, télégramme du 5 avril 1918, signé Dubos.

84. ANC, $1 \mathrm{~W} 1$, le gouverneur à M. le secrétaire général, nº 504, Nouméa, le 3 mai 1918, copie non signée; ANC, 23WC17, procureur général (Moreau), n 1242, le 30 novembre 1919.

85. Rapport du tribunal maritime spécial chargé des crimes commis par les condamnés en cours de peine, 25 mai 1918, cité in N. RATZEL, Cahiers..., op. cit., p. 39.

86. ANOM, FM, H/1 557, dossier de condamné, Mohamed ben Ahmed. Le ministre des Colonies au garde des Sceaux, 20 octobre 1921.

87. Ibidem, le garde des Sceaux au ministre des Colonies, 21 octobre 1933. 
en dépit des six relances faites par le garde des Sceaux au ministre des Colonies entre le 12 juin 1931 et le 15 octobre 1934. Le ministre des Colonies dit avoir répercuté auprès du gouverneur en juin 1931 sans succès et ne répond plus par la suite. Dans le même temps, deux notes découvertes dans les archives nous indiquent d'une part qu'il obtient la concession définitive du lot 202 à Pouembout en décembre 1932 et d'autre part que son fils Ahmed y est né au printemps de la même année; preuve que Mohamed ben Ahmed réside toujours à Pouembout et qu'il a reconstitué une famille ${ }^{88}$. Cependant, par une lettre de relance du 25 septembre 1934, le garde des Sceaux révèle que Mohamed ben Ahmed se trouve installé à Boghen sur la "commune mixte de Bourail "89. Situé à une centaine de kilomètres au sud de Koné, Boghen est une extension de Bourail, le centre de colonisation pénale le plus important du pays. Mohamed et sa famille semblent s'y être déplacés entre 1932 et 1934.

Sa demande de remise de résidence perpétuelle, quant à elle, est laissée en suspens lorsqu'intervient la Ligue des droits de l'Homme (LDH) à Paris, par une lettre du 24 octobre 1934 adressée au ministre des Colonies. Apparemment, ce n'est pas la première fois que la $\mathrm{LDH}$ alerte le ministre sur le cas de Mohamed:

«Nous avons déjà eu l'honneur d'appeler l'attention du ministère de la Justice sur la situation de Mohamed ben Ahmed qui se trouve actuellement à Boghen, commune mixte de Bourail [...]. Des explications qui nous sont fournies par la famille, il résulte que $M$. Ahmed a été condamné il y a environ trente-cinq ans aux travaux forcés à perpétuité pour meurtre par la cour d'assises d'Oran. [...] Nos correspondants nous indiquent que lors de l'insurrection des Canaques en 1917, il a réussi à capturer le chef, instigateur de cette insurrection; il obtint une prime de deux mille francs en récompense. Sa famille réside à Béni Saf, département d'Oran où elle est honorablement connue. Mohamed est libéré depuis plus de cinq ans; depuis qu'il est détenu, il n'a cessé d'avoir une bonne conduite; il est marié et père de quatre enfants ${ }^{90}$.

Cette lettre est étonnante à plus d'un titre. D'abord parce que la famille de Mohamed ben Ahmed semble se soucier de la situation d'un de ses membres parti d'Algérie depuis quarante ans tout juste, avec lequel on ignore si des liens ont pu être maintenus. On sait que Mohamed était orphelin de père lors de sa condamnation en 1889. On ignore s'il avait des frères et sœurs mais il avait une épouse et un enfant à son départ. Ses proches et peut-être son fils ou sa fille du premier lit se mettent visiblement à sa recherche en 1934 par l'intermédiaire de la LDH. Le fait est surprenant car Mohamed était issu d'un milieu de cultivateurs illettrés des zones frontalières du Maroc et de l'Algérie, lui-même pauvre journalier en 1889. Mais en 1934, la famille, installée à

88. ANC, service du Domaine, 730W-9, concession définitive du lot 202 à Pouembout; ANC, 465 W58, listes matricules des classes 52 (service militaire), naissance d'Ahmed Mohamed ben Ahmed.

89. Le terme est parfaitement incongru dans le contexte calédonien. Il est le fruit d'une confusion avec les "communes mixtes" algériennes, confusion à laquelle s'ajoute celle du numéro de matricule noté 13757 et non 13753 .

90. Ibidem, lettre de la Ligue française des droits de l'Homme et du citoyen adressée au ministre des Colonies, le 24 octobre 1934. 
Beni Saf, côté algérien, est "honorablement connue» et semble en mesure de s'adresser à la section de la Ligue à Alger puis à Paris. À moins que ce soit la Ligue des droits de l'Homme, à Paris, qui se soit mise en relation avec la famille car elle mène une campagne active dans les années 1930 en faveur de l'abolition de la transportation pénale et de la suppression du bagne en Guyane, et se trouve donc mobilisée sur ces questions, y compris celles qui touchent à la Nouvelle-Calédonie.

Cette lettre du 24 octobre 1934 est aussi étonnante parce qu'elle n'hésite pas à argumenter en revendiquant le seul "fait d'arme» de Mohamed ben Ahmed en 1917, à savoir le meurtre du chef Noël. Non sans contradiction avec son engagement, par ailleurs, en faveur des colonisés et contre les «abus" du système colonial, la Ligue y voit un acte positif pouvant favoriser une mesure de grâce pour le condamné dont elle loue par ailleurs la "bonne conduite» qu'il n'aurait cessé d'avoir depuis qu'il est détenu. Enfin, on apprend que Mohamed a refait sa vie en Nouvelle-Calédonie où il a une nouvelle épouse et quatre enfants en 1934 .

Comme le garde des Sceaux, la LDH se heurte au silence du gouverneur de la Nouvelle-Calédonie et doit insister par trois fois auprès du ministère des Colonies, en octobre et novembre 1934 et février 1935. Le 23 janvier 1935, le gouverneur réagit enfin en annonçant que Mohamed avait été placé dans le travail des grâces en 1934. Mal informé ou négligent, il affirme qu'il est en concession à Pouembout depuis 1916 sans aucune allusion à son passage à Koniambo et passe sous silence le fait qu'il réside désormais à Boghen. Le 18 avril 1935, le président de la République décide une remise de la résidence perpétuelle à trois ans pour le condamné Mohamed ben Ahmed, ce qui le rend libre de quitter la colonie en 1938. On perd sa trace à cette date et on ne sait quelle a été sa décision. Il semble y avoir, encore aujourd'hui, certains de ses descendants en Nouvelle-Calédonie.

Nous voici au terme d'une enquête passionnante, fruit d'un travail de longue haleine rendu ardu par le recoupement de nombreuses archives qu'il exigeait mais qui s'avère extrêmement fructueux. Car l'enquête, prenant appui sur la reconstitution aussi rigoureuse que possible de la trajectoire de Mohamed ben Ahmed dans le contexte colonial de la région de Koné au tournant du siècle, nous permet d'explorer un univers de relations et de pratiques qui jusque-là était resté opaque car difficile à documenter avec précision. L'examen mené au plus près du terrain confirme, si cela était nécessaire, le caractère redoutablement prédateur des logiques coloniales qui étendent progressivement sur les colonisés, en l'espèce les Kanak, un filet aux mailles de plus en plus serrées, réduisant leur espace vital tout en multipliant obligations et interdits. On mesure pour Noël et les siens ce que signifie en pratique "devenir indigènes" à Koniambo, exemplifiant des scènes malheureusement 
classiques de la «situation coloniale» telle qu'elle se déploie en Nouvelle-Calédonie comme dans les autres colonies françaises. La surprise vient ici des éclairages apportés sur les pressions qu'exercent le voisinage le plus proche, les colons les plus modestes et, parmi eux, certains condamnés aux travaux forcés placés sur concession de terre à l'issue de longues années de souffrance et que l'on peine même à désigner comme colons tant leur condition devrait rappeler celle des «subalternes».

La catégorie «subalterne» semble inappropriée lorsqu'elle s'applique aux familles Gros ou Fiacre qui forcent presque l'admiration par leur extraordinaire capacité à jouer de l'opportunité que leur offre la transportation pour se frayer une promotion sociale dans le pays d'exil et d'accueil, y compris en franchisant les frontières entre catégories pénales et libres que beaucoup, du côté des «libres" ou de l'administration, souhaiteraient maintenir étanches. Les indices signalant les distances ou proximités sociales dans la brousse calédonienne sont d'ailleurs difficiles à démêler car les fils Gros ou Fiacre peuvent avoir aussi de fortes connexions avec certains Kanak, pratiquer le "coup de pêche ou de chasse» et parler la langue du coin. Ce qui n'est pas le cas de certains colons installés au cœur des villages libres ou pénaux qui se refusent au contraire à toute "compromission", y compris à l'effort d'apprentissage linguistique représentant le risque de se "perdre" culturellement ou d'encanaquement comme on disait alors. La proximité de certains avec le monde kanak ne signifie pas qu'ils oublient à quelle catégorie de la population ils appartiennent lorsque leurs intérêts l'exigent - qu'il s'agisse de faire pression pour grignoter les terres de réserves ou d'exploiter les travailleurs. On voit ici toutes les nuances d'action et de positionnement qui se jouent dans la brousse calédonienne, à un niveau micro local, parmi des colons dont il faut souligner les origines sociales modestes pour la grande majorité, clivés par leur appartenance à la communauté «libre» ou "pénale» qui n'explique pas pour autant les variations des comportements face à l'Autre et, en tout premier lieu, le Kanak dans un monde local où les stratégies d'intérêts personnels restent essentielles. On remarquera que, contrairement à ce que voudrait faire prévaloir aujourd'hui l'histoire officielle, en ces temps de commémoration de la Grande Guerre, notre enquête nous amène aussi à questionner la vigueur de l'élan patriotique parmi certains colons qui, quoique français, œuvrent localement, y compris en instrumentalisant l'insurrection kanak, pour ne pas partir sur le front.

Mais revenons au cas de Mohamed ben Ahmed qui, plus que tout autre, brouille les cartes identitaires d'un monde colonial en formation. Originaire d'un Maroc qui n'est pas encore placé sous protectorat français mais tenu pour indigène lorsqu'il est arrêté en Algérie, il est mué en bagnard puis en concessionnaire pénal, processus qui le transforme en matricule et annihile finalement, en Nouvelle-Calédonie, son statut initial d'indigène. Néanmoins perçu comme "Arabe», Mohamed fait partie dans un premier temps des 
concessionnaires pénaux célibataires, sans ressources et sans défense comparés à un voisin tel que Fiacre. Sa condition subalterne lui permet peut-être de nouer plus aisément une relation étroite avec le Kanak Noël qui vient travailler sur sa concession, au point qu'il est soupçonné de soutenir les «insurgés» en 1917. Ceci ne l'empêche pas de le trahir et de revendiquer son geste auprès du gouverneur pour défendre sa cause et ses intérêts. Mohamed est-il un colonisé ou un colonisateur, un colon ou un indigène? Même la Ligue des droits de l'Homme semble s'y perdre en ne retenant de Mohamed que sa qualité de transporté, quitte à valoriser son crime en faveur d'une œuvre coloniale française dont la Ligue dénonce par ailleurs les "abus».

Une chose est sûre, c'est qu'en Nouvelle-Calédonie, la mémoire d'un tel personnage est aujourd'hui encore très difficile à évoquer. En 1977, un article publié à l'occasion du centenaire de la naissance du missionnaire Maurice Leenhardt reproduit une photo du chef Noël et précise qu'il «fut tué par traîtrise par un colon arabe, dont la légende veut qu'il soit mort peu après sans laisser de descendance ${ }^{91}$. Cette légende prend peut-être sa source dans un mystérieux "État des successions appréhendées du $1^{\text {er }}$ au 15 novembre 1919", publié dans le Fournal officiel de la Nouvelle Calédonie le 3 janvier 1920 et qui fait état d'un "Mohamed ben Ahmed» décédé le 30 septembre. L'année n'est pas précisée et il n'y a pas de numéro de matricule. Est-ce une erreur? Force est de constater que Mohamed ben Ahmed n'est pas mort en 1919. Mais peut-être a-t-on voulu le faire croire pour le faire disparaître.

Son souvenir a été à nouveau évoqué récemment en 2011, lors de l'exposition Caledoun: Arabes et Berbères de Nouvelle-Calédonie hier et aujourd'hui présentée pour la première fois à l'Institut du monde arabe à Paris. Un panneau explique que "ce transporté s'est pourtant rendu bien utile à l'administration coloniale en tuant le chef Noël pendant l'insurrection kanak de 1917 ». La formule est pour le moins ambiguë voire déplacée alors que s'organisent dans le même temps, en Nouvelle-Calédonie, des cérémonies kanak de réconciliation entre les clans descendants des insurgés et des auxiliaires indigènes de 1917, et que s'est tenue en 2009 à Koné, pour la première fois, une célébration coutumière en l'honneur de Noël. Celle-ci visait à conclure, enfin, les cérémonies de deuil et à élever une stèle en sa mémoire tout en fournissant l'occasion d'une rencontre pour libérer la parole et les souvenirs traumatiques de la guerre de 1917 en présence de tous les Kanak de la région, de membres des autres communautés et d'historiens et anthropologues invités. La stèle érigée en la mémoire de Noël a été placée sur le site même où il a été tué, l'ancienne concession de Mohamed ben Ahmed ou le lot 34 du centre pénal de Koniambo ${ }^{92}$.

91. J. GUIART, «Maurice Leenhardt inconnu: l'homme d'action», Objets et mondes. Revue du Musée de l'Homme - Muséum National d'Histoire Naturelle, 17-2, 1977, p. 75-84, p. 82.

92. A. Bensa, K.Y. Goromoedo, A. MUCKLe, Les sanglots..., op. cit., p. 182 et 184. 
En dépit de son crime, Mohamed ben Ahmed pourrait s'inscrire légitimement dans ce que Jean Marie Tjibaou appelait en 1985, dans son célèbre discours à Nainville-les-Roches, les "victimes de l'histoire», accordant à tous ceux que la France coloniale avait drainés de gré ou de force vers son pays une présence historique légitime. Si on peut comprendre qu'il soit encore très difficile d'évoquer la complexité de la trajectoire de Mohamed ben Ahmed en Nouvelle-Calédonie, on doit aussi défendre ici les enjeux heuristiques fondamentaux d'une démarche de connaissance historique qui cherche à restituer les traces du passé colonial dans sa globalité et sous l'angle le plus précis qui soit. La figure de Mohamed ben Ahmed illustre toute «l'ambiguïté» de la situation coloniale qu'évoquait Georges Balandier en 1951, et son itinéraire de vie replacé en contexte sert de levier pour relativiser la notion de «victimes de l'histoire" tout en rendant justice et responsabilité à tous dans un pays qui aspire à construire «un destin commun».

Isabelle MERLE Aix Marseille Université CNRS, EHESS, CREDO UMR 7308, 13331, Marseille France isabelle.merle13@gmail.com

Adrian MUCKLE Victoria University of Wellington PO Box 600

Wellington 6140

Nouvelle-Zélande adrian.muckle@vuw.ac.nz 


\section{Résumé / Abstract}

Isabelle MERLE, Adrian MUCKLE

Pour la mort d'un chef kanak. Le destin de Mohamed ben Ahmed ou les enjeux d'une histoire coloniale des subalternes. La Nouvelle-Calédonie au tournant du $\mathrm{xx}^{\mathrm{e}}$ siècle

Le chef kanak Bwëé Noël Pwatiba est mort, le 10 janvier 1918, au lieu-dit Koniambo, sous les coups d'un «arabe», condamné aux travaux forcés. C'est ainsi qu'était raconté en 2009, devant la stèle posée en son honneur, le décès de celui qui fut l'une des grandes figures de "la guerre de 17 ", nom donné aujourd'hui à l'insurrection kanak qui éclata dans le nord de la Grande Terre en Nouvelle-Calédonie dans l'ombre de la Grande Guerre. "L'arabe», Mohamed ben Ahmed, resté longtemps anonyme, est ici l'aiguillon de l'enquête qui tente de comprendre les raisons d'un crime. Il s'agit d'éclairer les plis et replis «d'un si petit monde colonial» où se côtoient des émigrants et des condamnés poussés par les dynamiques impériales du temps, à proximité de Kanak refoulés dans des réserves indigènes. Inspirés par une histoire au ras du sol prêtant une attention soutenue aux subalternes et à leurs logiques d'action, nous cherchons à rendre lisible la "situation coloniale" au plus près du terrain, à travers l'enchevêtrement de destins et de liens - ou du moins d'en comprendre l'ambiguïté à travers la rencontre improbable entre Mohamed ben Ahmed et le chef Noël, dans laquelle se jouent l'entraide, la fragile confiance et la trahison.

MotS-CLÉs: Océanie, $\mathrm{XX}^{\mathrm{e}}$ siècle, Nouvelle-Calédonie, situation coloniale, Middle ground, violence, colon, Kanak, indigènes

\section{Isabelle MERLE, Adrian MUCKLE}

For the death of a Kanak chief. The destiny of Mohamed ben Ahmed or a colonial history of subalterns in early-twentieth century New Caledonia

On Fanuary $10^{\text {th }} 1918$ the Kanak chief Bwëe Noël Pwatiba died at a place called Koniambo at the hands of an "Arab" convict sentenced to hard labour. In 2009, before the monument erected in his honour, it was in these terms that was recounted the death of one of the principal figures of "the war of 17", the name given today to the Kanak insurrection that broke out in the north of the Grande terre in the shadow of the Great War. The "Arab", Mohamed ben Ahmed, who has long remained in anonymity, is here the catalyst for the investigation which seeks to understand the reasons for this crime. We seek to shed light on the folds and pleats "of a very small colonial world" in which emigrants and convicts pushed by the imperial dynamics of the time rubbed shoulders with each other alongside Kanak driven into native reserves. Inspired by grounded histories that pay sustained attention to subalterns and the logics of their actions we seek to make readable the "colonial situation" at the grassroots level and the entanglement of destinies and connections. Or at the very least to understand the ambiguity of this situation through the improbable encounter between Mohamed ben Ahmed and the chief Noël in which mutual support, fragile trust and betrayal came into play.

KEYWORDS: Oceania, $20^{\text {th }}$ century, New Caledonia, colonial situation, Middle ground, violence, settler, Kanak, native 